\title{
Sensitivity of Photoneutron Production to Perturbations in Cross-Section Data
}

\section{October 2007}

\author{
Prepared by \\ Shaun D. Clarke \\ Sara A. Pozzi \\ Tom J. Downar \\ Enrico Padovani
}




\section{DOCUMENT AVAILABILITY}

Reports produced after January 1, 1996, are generally available free via the U.S. Department of Energy (DOE) Information Bridge.

Web site http://www.osti.gov/bridge

Reports produced before January 1, 1996, may be purchased by members of the public from the following source.

National Technical Information Service

5285 Port Royal Road

Springfield, VA 22161

Telephone 703-605-6000 (1-800-553-6847)

TDD 703-487-4639

Fax 703-605-6900

E-mail info@ntis.gov

Web site http://www.ntis.gov/support/ordernowabout.htm

Reports are available to DOE employees, DOE contractors, Energy Technology Data Exchange (ETDE) representatives, and International Nuclear Information System (INIS) representatives from the following source.

Office of Scientific and Technical Information

P.O. Box 62

Oak Ridge, TN 37831

Telephone 865-576-8401

Fax 865-576-5728

E-mail reports@osti.gov

Web site http://www.osti.gov/contact.html

This report was prepared as an account of work sponsored by an agency of the United States Government. Neither the United States government nor any agency thereof, nor any of their employees, makes any warranty, express or implied, or assumes any legal liability or responsibility for the accuracy, completeness, or usefulness of any information, apparatus, product, or process disclosed, or represents that its use would not infringe privately owned rights. Reference herein to any specific commercial product, process, or service by trade name, trademark, manufacturer, or otherwise, does not necessarily constitute or imply its endorsement, recommendation, or favoring by the United States Government or any agency thereof. The views and opinions of authors expressed herein do not necessarily state or reflect those of the United States Government or any agency thereof. 
Nuclear Science and Technology Division

\title{
SENSITIVITY OF PHOTONEUTRON PRODUCTION TO PERTURBATIONS IN CROSS-SECTION DATA
}

\author{
S.D. Clarke ${ }^{1,2 *}$, S. A. Pozzi, ${ }^{2}$, T.J. Downar ${ }^{3}$, and E. Padovani ${ }^{4}$ \\ ${ }^{1}$ School of Nuclear Engineering, Purdue University, West Lafayette, IN \\ ${ }^{2}$ Oak Ridge National Laboratory, Oak Ridge, TN \\ ${ }^{3}$ School of Nuclear Engineering, University of California, Berkeley, Berkeley, CA \\ ${ }^{4}$ Polytechnic of Milan, Milan, Italy
}

Date Published: October 2007

\author{
Prepared by \\ OAK RIDGE NATIONAL LABORATORY \\ P.O. Box 2008 \\ Oak Ridge, Tennessee 37831-6285 \\ managed by \\ UT-BATTELLE, LLC \\ for the \\ U.S. DEPARTMENT OF ENERGY \\ under contract DE-AC05-00OR22725
}

\footnotetext{
* On research assignment at Oak Ridge National Laboratory.
} 



\section{CONTENTS}

Page

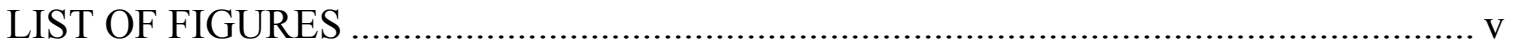

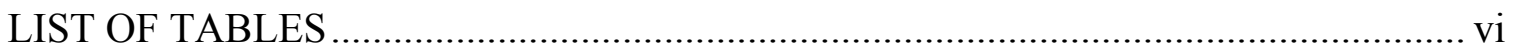

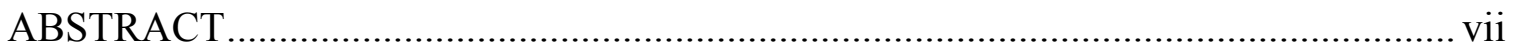

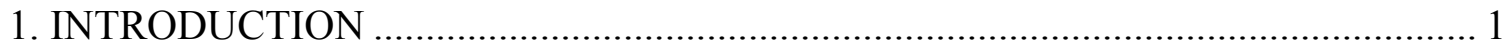

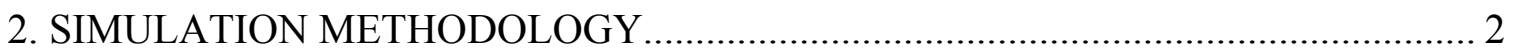

2.1 Description of Perturbation Techniques................................................................. 2

2.2 Description of the MCNP-PoliMi Model ............................................................ 3

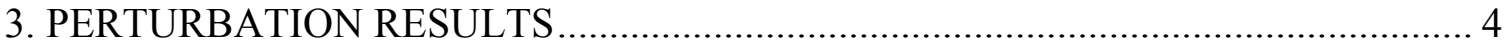

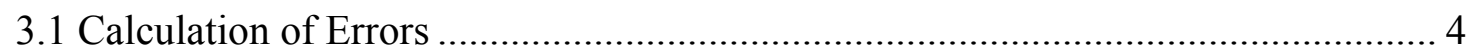

3.2 Photoneutron Production Results ...................................................................... 5

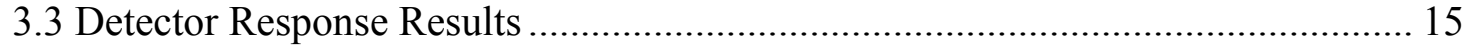

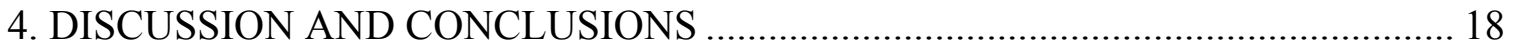

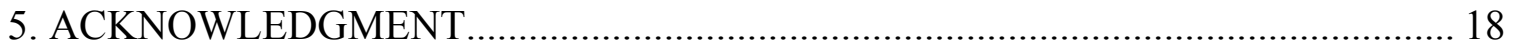

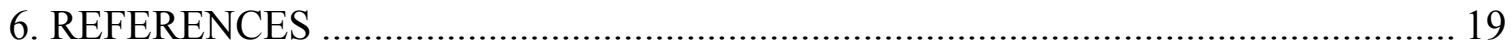

APPENDIX A. Monte Carlo Integration Routine ................................................. A-3

APPENDIX B. MCNP-PoliMi Input File.............................................................. B-3 



\section{LIST OF FIGURES}

\section{Figure}

1. Photonuclear reaction cross sections for ${ }^{238} \mathrm{U}$ from the ENDF/B-VII

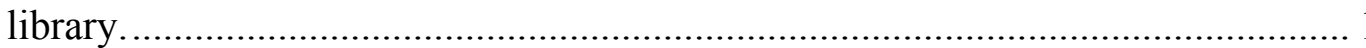

2. Calculation flow of the MCNPX/MCNP-PoliMi Code System. ........................... 2

3. MCNP-PoliMi model including the interrogation source, DU target and liquid scintillation detector.

4. $\quad$ Normalized photonuclear reaction cross sections for ${ }^{238} \mathrm{U}$............................... 5

5. Change in photoneutron production from a $10-\mathrm{MeV}$ beam following perturbations in the $(\gamma, n)$ and $(\gamma, 2 n)$ cross sections.

6. Change in average photoneutron energy from a $10-\mathrm{MeV}$ beam following perturbations in the $(\gamma, n)$ and $(\gamma, 2 n)$ cross sections.

7. Change in photoneutron production from a $15-\mathrm{MeV}$ beam following perturbations in the $(\gamma, n)$ and $(\gamma, 2 n)$ cross sections.

8. Change in average photoneutron energy from a $15-\mathrm{MeV}$ beam following perturbations in the $(\gamma, n)$ and $(\gamma, 2 n)$ cross sections.

9. Change in photoneutron production from a $20-\mathrm{MeV}$ beam following perturbations in the $(\gamma, n)$ and $(\gamma, 2 n)$ cross sections.

10. Change in average photoneutron energy from a $20-\mathrm{MeV}$ beam following perturbations in the $(\gamma, n)$ and $(\gamma, 2 n)$ cross sections.

11. Simulated time-of-flight spectrum generated with a $10-\mathrm{MeV}$ beam and a 0.0793-MeVee detection threshold 


\section{LIST OF TABLES}

\section{Table}

1. Photoneutron production from a $10-\mathrm{MeV}$ beam following perturbations of the $(\gamma, n)$ and $(\gamma, 2 n)$ cross sections; the relative difference to the base case is also shown.

2. Photoneutron production from a $15-\mathrm{MeV}$ beam following perturbations of the $(\gamma, n)$ and $(\gamma, 2 n)$ cross sections; the relative difference to the base case is also shown.

3. Photoneutron production from a $20-\mathrm{MeV}$ beam following perturbations of the $(\gamma, n)$ and $(\gamma, 2 n)$ cross sections; the relative difference to the base case is also shown.

4. Relative errors $(1-\sigma)$ of photoneutron production from a $10-\mathrm{MeV}$ beam following perturbations of the $(\gamma, n)$ and $(\gamma, 2 n)$ cross sections; mean values are listed in Table 1

5. Relative errors $(1-\sigma)$ of photoneutron production from a $15-\mathrm{MeV}$ beam following perturbations of the $(\gamma, n)$ and $(\gamma, 2 n)$ cross sections; mean values are listed in Table 2 .

6. Relative errors $(1-\sigma)$ of photoneutron production from a $20-\mathrm{MeV}$ beam following perturbations of the $(\gamma, n)$ and $(\gamma, 2 n)$ cross sections; mean values are listed in Table 3 ....

7. Photoneutron production sensitivity with respect to perturbations in the $(\gamma, n)$ reaction cross section $\left(S_{1}\right)$; the $1-\sigma$ relative error is also shown. 15

8. Photoneutron production sensitivity with respect to perturbations in the $(\gamma, 2 n)$ reaction cross section $\left(S_{2}\right)$; the $1-\sigma$ relative error is also shown.

9. MCNP-PoliMi time-integrated neutron counts from a $10-\mathrm{MeV}$ beam; relative difference to the base case is also shown.

10. MCNP-PoliMi time-integrated neutron counts from a $15-\mathrm{MeV}$ beam; relative difference to the base case is also shown.

11. MCNP-PoliMi time-integrated neutron counts from a 20-MeV beam; relative difference to the base case is also shown. 


\begin{abstract}
The most recent release of photonuclear interaction data for Monte Carlo applications is the ENDF/B-VII library. While this current version offers several improvements over its predecessors, it does not address the observed, sometimes quite significant variance in the measured data. For instance, for ${ }^{238} \mathrm{U}$, the cross-section data in the ENDF/B-VII library is consistently larger than all measurements except for those by Caldwell, et al., occasionally by as much as $20 \%$. The objective of the work performed here was to investigate the sensitivity of photoneutron production to perturbations in photonuclear cross-section data. The effect of these perturbations on experimental observables in a common setup was assessed using the MCNPX/MCNP-PoliMi code system. Since the standard MCNPX perturbation routines are not available for photonuclear reactions, we developed and implemented a new methodology to evaluate the sensitivity of commonlymeasured parameters to perturbations in photonuclear cross-section data. The results of the analysis show that the maximum variance applied to the cross section $(20 \%)$ results in an integral detector response change that in general varies between $4 \%$ and $8 \%$ for the exact configuration considered here. However, the methodology is general and may be readily applied to any source-target configuration. This has direct implications on the design of active interrogation systems for nuclear nonproliferation and homeland security applications as well as to any other applications using photonuclear cross-section data.
\end{abstract}





\section{INTRODUCTION}

Systems relying on active interrogation are gaining more attention from the research community in the areas of nuclear nonproliferation and homeland security. One approach utilizes high-energy bremsstrahlung photons to induce photofission and other photonuclear reactions in the material of interest. The emissions from these reactions are then detected and used to characterize the material. Because these measurement scenarios can be large and complex, the Monte Carlo method is the most widely applied approach to their design and analysis. In particular, the MCNPX/MCNP-PoliMi code system has been developed to simulate all aspects of these systems - from the source electrons to the final correlated detector response. However, all simulation tools are limited by the quality of initial nuclear data, and there are clear inconsistencies in the literature reporting photonuclear interaction data. In fact, recent publications show variances as large as $20 \%$ between various measurements of the $(\gamma, n)$, and $(\gamma, 2 n)$ cross-section data [1,2].

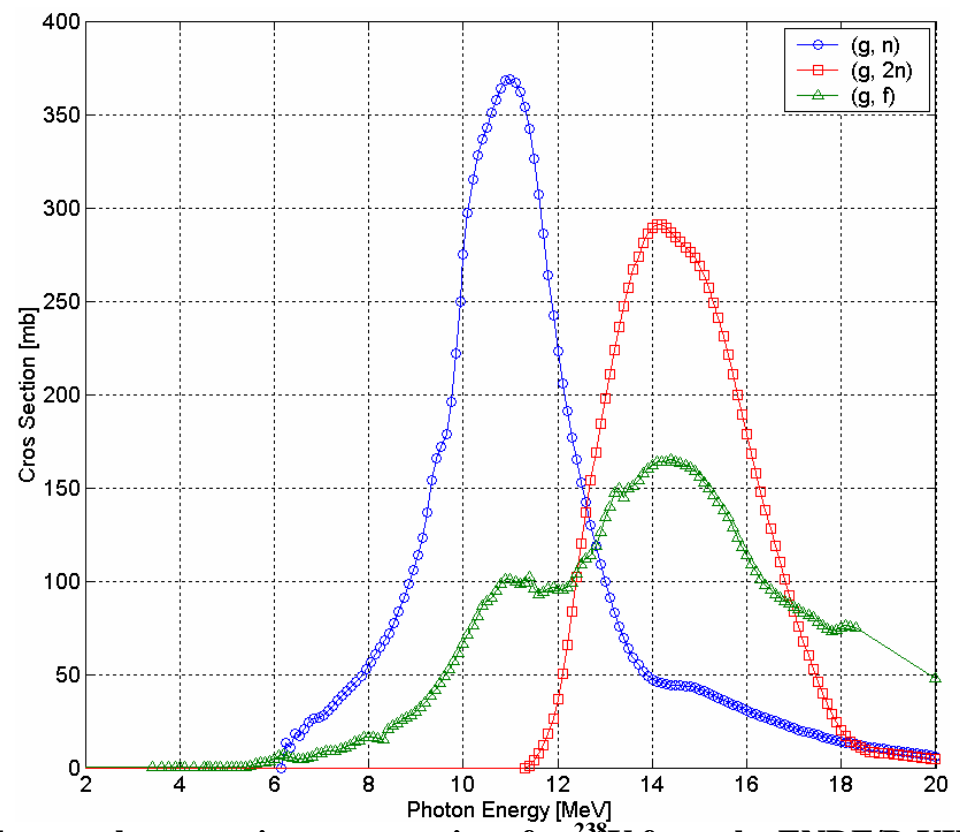

Fig. 1. Photonuclear reaction cross sections for ${ }^{238} U$ from the ENDF/B-VII library.

The most recent release of photonuclear interaction data is the ENDF/B-VII libraryshown in Fig. 1, offers several improvements over its predecessors, but it does not address the observed, sometimes quite significant variance in the measured data. For example, the ${ }^{238} \mathrm{U}$, the cross-section data in the ENDF/B-VII library is consistently larger than all measurements except for those by Caldwell, et al. [3], occasionally by as much as $20 \%$. The objective of the work performed here was to investigate the sensitivity of photoneutron production to perturbations in photonuclear cross-section data and to estimate the uncertainty in the simulation of typical experiments introduced by the uncertainty in the photoneutron cross section data. The proposed methodology is applied 
to a depleted uranium (DU) target. However, it can be easily extended to other target materials.

\section{SIMULATION METHODOLOGY}

The MCNPX/MCNP-PoliMi code was used for this analysis in order to obtain the most accurate simulation of the detector response. MCNP-PoliMi version 1.2.4 is capable of running with all standard MCNP source types and includes several specific spontaneousfission-source definitions (i.e., ${ }^{252} \mathrm{Cf},{ }^{240} \mathrm{Pu},{ }^{242} \mathrm{Pu},{ }^{242} \mathrm{Cm},{ }^{244} \mathrm{Cm}$ ), as well as Am-Li and Am-Be isotopic sources. In addition, a photonuclear source file may be generated using a modified version of MCNPX and read by MCNP-PoliMi [4].

\subsection{Description of Perturbation Techniques}

The standard MCNPX perturbation routines are not available for photonuclear reactions [5]. Therefore, a new methodology has been developed and implemented that utilizes the modular nature of the MCNPX/MCNP-PoliMi code system. Fig. 2 illustrates the calculation flow of a MCNP-PoliMi simulation with a photonuclear source. A modified version of MCNPX is used to transport the source photons to the target. The results of all photonuclear reactions that occur in the target are written to a "photonuclear reaction source file." The contents of this file include the energy of the interacting photon, the target isotope, the reaction type, and its geometric location. In the case of a simple photoneutron reaction - $(\gamma, n),(\gamma, 2 n)$, or $(\gamma, 3 n)$ - the reaction products, and their energy, are also written to the file. In the case of $(\gamma$, fission), the reaction products are not written to the file. Instead, only the $Q$-value is recorded; MCNP-PoliMi generates the fission neutrons and gamma rays - along with number, energy and direction - using full, or correct, multiplicity distributions.

The reaction source file is read by MCNP-PoliMi which then transports the products of each source event through geometry. The energy released during each collision in the detectors, the corresponding time, the incident particle type, and the target nucleus are saved in a "collision output file." A post-processing code is then used to load the required data from this file and compute the detector-specific response.

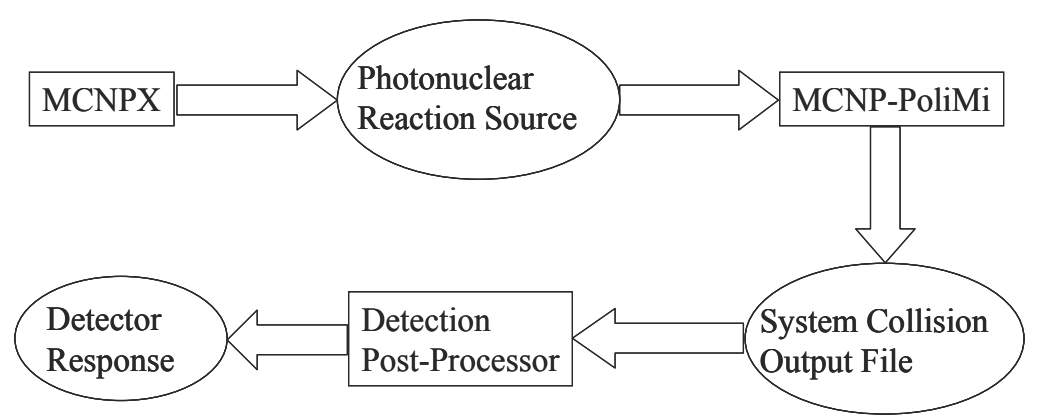

Fig. 2. Calculation flow of the MCNPX/MCNP-PoliMi Code System. 
In order to apply the desired perturbations, an external calculation is performed after the initial MCNPX simulation. More precisely, the source bremsstrahlung spectrum is integrated with the perturbed photonuclear cross sections to calculate the resulting reaction rates. This integration is performed using a Monte Carlo technique in order to preserve the spatial effect of the finite target dimensions. The integration routine is written in the MATLAB program language.

1. The full photonuclear cross-section set is loaded and the data are perturbed by the desired amount.

2. At the start of each history the initial photon energy is sampled from a bremsstrahlung spectrum.

3. The partial, and total, photonuclear cross sections are computed at the photon energy.

4. The photonuclear-interaction distance is sampled.

5. If the photonuclear-interaction distance is less than the target thickness, the photon interacts.

6. The interaction type is sampled from the partial cross sections.

7. The appropriate number of neutrons is generated and banked.

In the case of fission induced by a photon of energy $E_{\gamma}$, the average number of neutrons emitted is computed from the following empirical relationship [6]:

$$
\bar{v}=1.862+0.123 E_{\gamma} .
$$

This process is repeated for the desired number of histories. Afterwards the number and type of all reactions that occur is tallied along with the corresponding statistical error. A perturbed photonuclear reaction source file is constructed using the partial reaction rates.

The unperturbed (base-case) file is divided into three separate files each containing all of the data for each reaction type; the perturbed file is written using the data from these individual files. The number of reaction entries to be written in the perturbed file is computed from the relative number of reactions in the perturbed case relative to the base case. For each reaction entry to be written, a random number is sampled and compared to the partial reaction rates; this determines from which partial data file each entry is read and written to the perturbed source file.

\subsection{Description of the MCNP-PoliMi Model}

The perturbed photonuclear source files were loaded by MCNP-PoliMi to tally the desired observables (number of neutrons crossing the detector face, average energy, and detector response). Sources of 10, 15, and 20-MeV-bremsstrahlung photons were simulated with a DU target placed $2 \mathrm{~m}$ from a $50 \times 50-\mathrm{cm}$, lead-shielded liquid

scintillation detector. This system, shown in Fig. 3, is representative of current active 
interrogation systems currently under investigation for nonproliferation and homeland security applications.

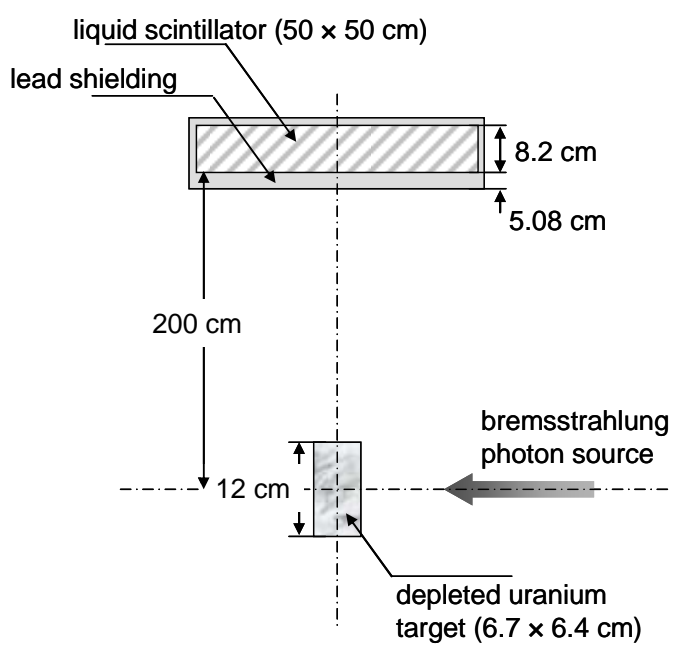

Fig. 3. MCNP-PoliMi model including the interrogation source, DU target, and liquid scintillation detector.

\section{PERTURBATION RESULTS}

Photoneutron production was analyzed under $(\gamma, n)$ and $(\gamma, 2 n)$ cross-section perturbations of $-0.05,-0.10,-0.15$ and -0.20 (the $(\gamma, f)$ cross section was held constant) for 10,15 and $20-\mathrm{MeV}$ bremsstrahlung photon sources. These specific perturbations were chosen because the photofission experimental results in the literature agree very well with one another while the $(\gamma, n)$ and $(\gamma, 2 n)$ results differ by as much as $20 \%[1,2]$.

\subsection{Calculation of Errors}

All quantities computed with the Monte Carlo method have an inherent statistical error. When performing operations with these quantities, these errors propagate through to the calculated values according to the following relationship [7]:

$$
\sigma_{u}^{2}=\left(\frac{\partial u}{\partial x}\right)^{2} \sigma_{x}^{2}+\left(\frac{\partial u}{\partial y}\right)^{2} \sigma_{y}^{2}+\left(\frac{\partial u}{\partial z}\right)^{2} \sigma_{z}^{2}+\ldots
$$

where $u(x, y, z, \ldots)$ is computed from the quantities $x, y, z, \ldots$ and $\sigma_{i}$ is the associated error. For the applications of interest, addition and multiplication are the two operations of primary concern. For addition or subtraction of two quantities, $x$ and $y$, Eq. 2 reduces to, 


$$
\sigma_{u}=\sqrt{\sigma_{x}^{2}+\sigma_{y}^{2}}
$$

For multiplication or division of two quantities, $x$ and $y$, Eq. 2 reduces to,

$$
\sigma_{u}=u \sqrt{\left(\frac{\sigma_{x}}{x}\right)^{2}+\left(\frac{\sigma_{y}}{y}\right)^{2}} .
$$

The statistical errors associated with each Monte Carlo tallied quantity were propagated using these relationships. For example, the fraction of $(\gamma, n)$ reactions is equal to the total number of $(\gamma, n)$ reactions tallied divided by the total number of reactions tallied. Each of these tallied quantities has a statistical error that is propagated using Eq. 4.

\subsection{Photoneutron Production Results}

Table 1 - Table 3 summarize the results of these calculations with a 10,15 and $20-\mathrm{MeV}$ beams. The number of reactions per source photon, $\left\langle N_{r}\right\rangle$, and the partial contribution from each individual reaction, $f_{(\gamma, n)}, f_{(\gamma, 2 n)}$, and $f_{(\gamma, f)}$, were computed using the Monte Carlo integration described earlier. The average number of neutrons per source photon, $\left\langle N_{n}\right\rangle$, and their average energy, $\left\langle E_{n}\right\rangle$ crossing the faces of the target and detector were computed with MCNP-PoliMi, (denoted by superscripts $t$ and $d$ respectively). The statistical error for each of these quantities is shown in Tables 4 through 6 .

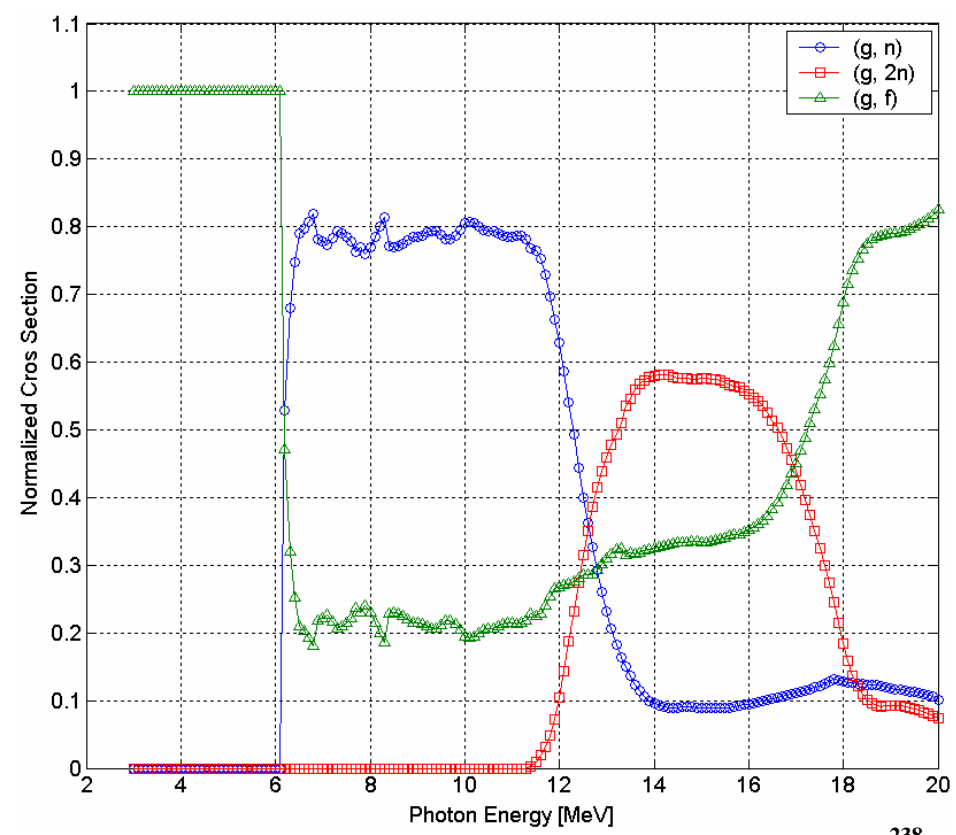

Fig. 4. Normalized photonuclear reaction cross sections for ${ }^{238} U$. 
Table 1. Photoneutron production from a 10-MeV beam following perturbations of the $(\gamma, n)$ and $(\gamma, 2 n)$ cross sections; the relative difference to the base case is also shown.

\begin{tabular}{|c|c|c|c|c|c|c|c|c|c|}
\hline$(\gamma, \mathrm{n}$ & $\delta_{t}$ & $f_{(\gamma, n)}$ & $f_{(y, 2 n)}$ & $f_{(\gamma, f)}$ & $<N_{r}>$ & $<N_{n}^{t}>$ & $<E_{n}^{t}>$ & $<N_{n}^{d}>$ & $<E_{n}^{d}>$ \\
\hline \multirow{9}{*}{0.00} & 0.00 & $\begin{array}{c}0.7654 \\
---\end{array}$ & 0.0000 & $\begin{array}{c}0.2346 \\
---\end{array}$ & $\begin{array}{c}9.276 \mathrm{E}-04 \\
---\end{array}$ & $\begin{array}{c}1.366 \mathrm{E}-03 \\
---\end{array}$ & $\begin{array}{c}1.137 \mathrm{E}+00 \\
--\end{array}$ & 2.932E-06 & $\begin{array}{c}9.514 \mathrm{E}-01 \\
---\end{array}$ \\
\hline & \multirow{2}{*}{-0.05} & 0.7637 & 0.0000 & 0.2363 & 9.213E-04 & 1.344E-03 & $1.129 \mathrm{E}+00$ & 2.885E-06 & 9.485E-01 \\
\hline & & -0.0022 & --- & 0.0072 & -0.0068 & -0.0158 & -0.0077 & -0.0162 & -0.0030 \\
\hline & \multirow{2}{*}{-0.10} & 0.7645 & 0.0000 & 0.2356 & 9.382E-04 & 1.378E-03 & $1.135 E+00$ & 2.960E-06 & 9.485E-01 \\
\hline & & -0.0013 & --- & 0.0041 & 0.0114 & 0.0089 & -0.0018 & 0.0093 & -0.0030 \\
\hline & \multirow{2}{*}{-0.15} & 0.7630 & 0.0000 & 0.2370 & $9.356 \mathrm{E}-04$ & 1.378E-03 & $1.138 \mathrm{E}+00$ & $2.944 \mathrm{E}-06$ & 9.541E-01 \\
\hline & & -0.0032 & --- & 0.0104 & 0.0087 & 0.0089 & 0.0004 & 0.0040 & 0.0028 \\
\hline & \multirow{2}{*}{-0.20} & 0.7621 & 0.0000 & 0.2379 & $9.396 \mathrm{E}-04$ & 1.369E-03 & $1.127 \mathrm{E}+00$ & 2.930E-06 & $9.469 \mathrm{E}-01$ \\
\hline & & -0.0043 & --- & 0.0142 & 0.0130 & 0.0026 & -0.0086 & -0.0007 & -0.0047 \\
\hline \multirow{10}{*}{-0.05} & \multirow{2}{*}{0.00} & 0.7573 & 0.0000 & 0.2427 & $8.938 \mathrm{E}-04$ & $1.326 \mathrm{E}-03$ & $1.145 E+00$ & $2.854 \mathrm{E}-06$ & 9.615E-01 \\
\hline & & -0.0106 & --- & 0.0346 & -0.0364 & -0.0290 & 0.0068 & -0.0266 & 0.0106 \\
\hline & \multirow{2}{*}{-0.05} & 0.7601 & 0.0000 & 0.2399 & $9.002 \mathrm{E}-04$ & 1.343E-03 & $1.150 \mathrm{E}+00$ & 2.882E-06 & 9.624E-01 \\
\hline & & -0.0069 & --- & 0.0226 & -0.0295 & -0.0170 & 0.0113 & -0.0172 & 0.0116 \\
\hline & \multirow{2}{*}{-0.10} & 0.7568 & 0.0000 & 0.2432 & 9.056E-04 & $1.351 \mathrm{E}-03$ & $1.150 \mathrm{E}+00$ & 2.893E-06 & $9.662 \mathrm{E}-01$ \\
\hline & & -0.0113 & --- & 0.0367 & -0.0237 & -0.0108 & 0.0112 & -0.0134 & 0.0155 \\
\hline & \multirow{2}{*}{-0.15} & 0.7557 & 0.0000 & 0.2443 & 9.009E-04 & 1.343E-03 & $1.149 E+00$ & 2.874E-06 & 9.627E-01 \\
\hline & & -0.0127 & --- & 0.0416 & -0.0288 & -0.0167 & 0.0107 & -0.0199 & 0.0119 \\
\hline & \multirow{2}{*}{-0.20} & 0.7552 & 0.0000 & 0.2448 & 9.014E-04 & 1.350E-03 & $1.154 \mathrm{E}+00$ & 2.893E-06 & 9.674E-01 \\
\hline & & -0.0134 & --- & 0.0437 & -0.0283 & -0.0114 & 0.0147 & -0.0132 & 0.0168 \\
\hline \multirow{10}{*}{-0.10} & \multirow{2}{*}{0.00} & 0.7448 & 0.0000 & 0.2552 & 8.661E-04 & 1.314E-03 & $1.166 \mathrm{E}+00$ & 2.822E-06 & $9.734 \mathrm{E}-01$ \\
\hline & & -0.0269 & --- & 0.0878 & -0.0663 & -0.0379 & 0.0251 & -0.0376 & 0.0231 \\
\hline & \multirow{2}{*}{-0.05} & 0.7460 & 0.0000 & 0.2540 & 8.655E-04 & 1.294E-03 & $1.152 \mathrm{E}+00$ & $2.788 \mathrm{E}-06$ & $9.639 \mathrm{E}-01$ \\
\hline & & -0.0253 & --- & 0.0826 & -0.0670 & -0.0526 & 0.0130 & -0.0491 & 0.0131 \\
\hline & \multirow{2}{*}{-0.10} & 0.7464 & 0.0000 & 0.2536 & 8.634E-04 & 1.298E-03 & $1.157 \mathrm{E}+00$ & 2.788E-06 & $.708 E-01$ \\
\hline & & -0.0248 & --- & 0.0811 & -0.0692 & -0.0496 & 0.0177 & -0.0492 & 0.02 \\
\hline & \multirow{2}{*}{-0.15} & 0.7418 & 0.0000 & 0.2582 & 8.634E-04 & 1.302E-03 & $1.160 \mathrm{E}+00$ & 2.804E-06 & $9.763 \mathrm{E}-01$ \\
\hline & & -0.0309 & --- & 0.1007 & -0.0693 & -0.0468 & 0.0203 & -0.0436 & 0.0262 \\
\hline & \multirow{2}{*}{-0.20} & 0.7410 & 0.0000 & 0.2590 & 8.726E-04 & 1.311E-03 & $1.157 \mathrm{E}+00$ & 2.832E-06 & 9.672E-01 \\
\hline & & -0.0319 & --- & 0.1041 & & -0.0401 & 0.0170 & -0.0341 & 0.0166 \\
\hline \multirow{10}{*}{-0.15} & & 0.7359 & 0.0000 & 0.2641 & 8.324E-04 & 1.273E-03 & $1.173 \mathrm{E}+00$ & 2.75 & 9.807E-01 \\
\hline & 0.00 & -0.03 & --- & 0.1259 & & & & & \\
\hline & & 0.7306 & 0.0000 & 0.2694 & 1E-04 & 1.280E-03 & $3 E+00$ & 2.75 & $=-01$ \\
\hline & & -0.0456 & --- & 0.1486 & 29 & -0.0631 & & -0.0 & 0.0 \\
\hline & -0.10 & 0.7374 & 0.0000 & 0.2626 & 8.191E-04 & $1.238 \mathrm{E}-03$ & $1.162 \mathrm{E}+00$ & 2.662E-06 & $9.709 \mathrm{E}-01$ \\
\hline & & & --- & & & & & & \\
\hline & & 0.7335 & 0.0000 & 0.2665 & E-04 & 1.264E-03 & 1.176 & 2.730E-06 & $9.829 \mathrm{E}-01$ \\
\hline & & -0.0417 & --- & 0.13 & & -0.07 & & & 0.03 \\
\hline & 20 & 0.7309 & 0.0000 & 0.2691 & 8.304E-04 & 1.264E-03 & $1.169 \mathrm{E}+00$ & 2.723E-06 & 9.785E-01 \\
\hline & $-0 . \angle 0$ & -0.0451 & --- & & & & & & \\
\hline & & 0.7221 & 0.0000 & 0.2779 & E-04 & 1.240E-03 & $1.188 \mathrm{E}+00$ & 2.67 & 9.899 \\
\hline & & & --- & 0.18 & & -0.091 & & & \\
\hline & & 0.7193 & 0.0000 & 0.2807 & 7.920E-04 & $1.233 \mathrm{E}-03$ & $1.189 E+00$ & 2.656E-06 & 9.964E-01 \\
\hline & & -0.0602 & --- & 0.1966 & -0.1461 & -0.0972 & 0.0459 & -0.0941 & 0.0473 \\
\hline-0.20 & .10 & 0.7210 & 0.0000 & 0.2790 & 7.898E-04 & 1.229E-03 & $1.189 E+00$ & 2.648E-06 & 9.914E-01 \\
\hline-0.20 & & -0.0581 & --- & 0.1896 & & & & & \\
\hline & & 0.7181 & 0.0000 & 0.2819 & 7.940E-04 & $1.238 \mathrm{E}-03$ & $1.191 \mathrm{E}+00$ & 2.679E-06 & 9.947E-01 \\
\hline & & -0.0619 & --- & 0.2019 & & & & -0.0 & \\
\hline & & 0.7322 & 0.0000 & 0.2678 & 8.049E-04 & 1.247E-03 & $1.185 E+00$ & 2.699E-06 & $9.888 \mathrm{E}-01$ \\
\hline & & & & 0.1415 & -0.13 & -0.0873 & 0.0418 & -0.0796 & 0.0394 \\
\hline
\end{tabular}


Table 2. Photoneutron production from a 15-MeV beam following perturbations of the $(\gamma, n)$ and $(\gamma, 2 n)$ cross sections; the relative difference to the base case is also shown.

\begin{tabular}{|c|c|c|c|c|c|c|c|c|c|}
\hline , n) & 2n) & $f_{(\gamma, n)}$ & $\gamma, 2 n)$ & & $<N_{r}>$ & $<N_{n}^{t}>$ & $<E_{n}^{t}>$ & $<N_{n}^{d}>$ & $<E_{n}^{d}>$ \\
\hline \multirow{9}{*}{0.00} & 0.00 & 0.6207 & 0.1333 & 0.2460 & $\begin{array}{c}4.283 \mathrm{E}-03 \\
---\end{array}$ & $\begin{array}{c}\text { 7.364E-03 } \\
---\end{array}$ & $\begin{array}{c}1.244 \mathrm{E}+00 \\
---\end{array}$ & $\begin{array}{c}1.605 \mathrm{E}-05 \\
---\end{array}$ & 030E+00 \\
\hline & \multirow{2}{*}{-0.05} & 0.6247 & 0.1289 & 0.2464 & $4.236 \mathrm{E}-03$ & 7.338E-03 & $1.250 \mathrm{E}+00$ & $1.604 \mathrm{E}-05$ & $1.030 \mathrm{E}+00$ \\
\hline & & 0.0065 & -0.0333 & 0.0017 & -0.0110 & -0.0036 & 0.0043 & -0.0008 & -0.0003 \\
\hline & \multirow{2}{*}{-0.10} & 0.6268 & & & 4.243E-03 & 7.321E-03 & $1.249 E+00$ & & $1.034 \mathrm{E}+00$ \\
\hline & & 0.0098 & -0.0907 & 0.0243 & -0.0094 & -0.0059 & 0.0039 & -0.0057 & 0.0040 \\
\hline & \multirow{2}{*}{-0.15} & 0.6337 & 0.1148 & 0.2515 & 4.209E-03 & $7.225 \mathrm{E}-03$ & $1.252 \mathrm{E}+00$ & $1.575 \mathrm{E}-05$ & $1.031 \mathrm{E}+00$ \\
\hline & & 0.0210 & -0.1391 & 0.0224 & -0.0173 & -0.0189 & 0.0059 & -0.0186 & 0.0004 \\
\hline & \multirow{2}{*}{-0.20} & 0.6359 & 0.1097 & 0.2543 & 4.152E-03 & 7.157E-03 & $1.259 E+00$ & 1.560E-05 & $1.039 E+00$ \\
\hline & & 0.0245 & -0.1770 & 0.0340 & -0.0305 & -0.0281 & 0.0121 & -0.0281 & 0.0085 \\
\hline \multirow{10}{*}{-0.05} & \multirow{2}{*}{0.00} & 0.6098 & 0.1368 & 0.2534 & 4.151 & 7.313E-03 & $1.248 \mathrm{E}+00$ & 1.593E-05 & $1.032 \mathrm{E}+00$ \\
\hline & & .0176 & 0.0261 & 0.0302 & -0.0 & -0.0069 & 0.0027 & & 0.0017 \\
\hline & \multirow{2}{*}{-0.05} & 0.6103 & & 0.2577 & 4.126 & 7.275E-03 & $1.258 \mathrm{E}+00$ & $1.587 \mathrm{E}-05$ & $1.039 \mathrm{E}+00$ \\
\hline & & -0.0167 & -0.0105 & 0.0478 & -0.03 & -0.0121 & 0.0114 & -0.0108 & 0.0083 \\
\hline & \multirow{2}{*}{-0.10} & 0.6160 & 0.1255 & 0.2584 & 4.101 & $7.216 \mathrm{E}-03$ & $1.263 \mathrm{E}+00$ & 1.570E-05 & $1.043 E+00$ \\
\hline & & -0.0075 & & 0.0505 & -0.0 & -0.0202 & 0.0150 & -0.0217 & 0.0124 \\
\hline & \multirow{2}{*}{-0.15} & 0.6212 & & & 4.099 & 7.180E-03 & 1.262 & $=-05$ & $1.042 \mathrm{E}+00$ \\
\hline & & 0.0007 & -0.0961 & 0.0502 & -0.04 & -0.0250 & 0.0142 & & 0.0114 \\
\hline & \multirow{2}{*}{-0.20} & 0.6277 & & 0.25 & 4.04 & 7.04 & 1.268 & 1.54 & $1.042 \mathrm{E}+00$ \\
\hline & & 0.0112 & & & & & & & \\
\hline \multirow{10}{*}{-0.10} & \multirow{2}{*}{0.00} & 0.5970 & 0.1434 & 2596 & 4.035 & 7.163E-03 & $1.258 \mathrm{E}+00$ & 1.561E-05 & $1.038 \mathrm{E}+00$ \\
\hline & & -0.0382 & & 0554 & $-0 . c$ & -0.0274 & 0.01 & -0.0273 & 0.0079 \\
\hline & \multirow{2}{*}{-0.05} & 0.5993 & 0.1350 & 2657 & 3.993 & 7.087E-03 & 1.262 & $1.548 \mathrm{E}-05$ & $1.044 \mathrm{E}+00$ \\
\hline & & -0.0345 & & & & & & & \\
\hline & \multirow{2}{*}{-0.10} & & & 2627 & 3.992 & 7.11 & $1.269 \mathrm{l}$ & E-05 & \\
\hline & & -0.0222 & -0.02 & 0.0678 & & & & & \\
\hline & \multirow{2}{*}{-0.15} & 0.6080 & & 2673 & 3.95 & 6.9 & $1.267 \mathrm{l}$ & 1.526 & $1.048 \mathrm{E}+00$ \\
\hline & & .0204 & & 0866 & & -0.0502 & & $-0.04 \varepsilon$ & 3 \\
\hline & \multirow{2}{*}{-0.20} & 0.6117 & 0.117 & 2709 & 3.943 & 6.996E-03 & $1.273 \mathrm{E}$ & E-05 & $1.049 \mathrm{E}+00$ \\
\hline & & .0144 & & & & & & & \\
\hline \multirow{10}{*}{-0.15} & & 300 & & 2716 & 3.89 & 7.0 & $1.264 \mathrm{E}$ & E-05 & $1.043 \mathrm{E}+00$ \\
\hline & & & & & & & & & \\
\hline & & 0.5 & & & 3.86 & 6.9 & 1.27 & 1.52 & 1.0 \\
\hline & & & & & & & & & \\
\hline & & 0.5900 & 0.1350 & 2750 & 3.82 & 6.92 & $1.269 \mathrm{E}+00$ & $1.513 \mathrm{E}-05$ & $1.050 \mathrm{E}+00$ \\
\hline & & & & & & & & & \\
\hline & & & & & & & 1.27 & & $1.05 c$ \\
\hline & & & & & & & & & \\
\hline & & 0.6003 & 0.1213 & 2785 & 3.78 & $6.7 \varsigma$ & $1.280 \mathrm{E}+00$ & 1.48 & $1.055 E+00$ \\
\hline & & & & & & & & & \\
\hline & & & & & 3.7 & -03 & 1.27 & & 1.05 \\
\hline & & & & & & & & & \\
\hline & & 0.5728 & 0.146 & 2809 & $3.734 \mathrm{E}-03$ & $6.868 \mathrm{E}-03$ & $1.281 \mathrm{E}+00$ & 1.509E-05 & $1.055 \mathrm{E}+00$ \\
\hline & & & & & & & & -0.0598 & 0.0238 \\
\hline & 10 & 0.5783 & 0.1406 & 0.2811 & 3.709E-03 & $6.821 \mathrm{E}-03$ & $1.282 \mathrm{E}+00$ & 1.499E-05 & $1.054 \mathrm{E}+00$ \\
\hline & & -0.0684 & & & & & & & \\
\hline & & 0.5780 & & 0.2873 & 3.689 & $6.755 \mathrm{E}-03$ & $1.286 \mathrm{t}$ & 1.475E-05 & $1.062 \mathrm{E}+00$ \\
\hline & & & & & & & & & \\
\hline & & & & & 3.673 & 6.707E-03 & $1.291 \mathrm{E}$ & 1.470E-05 & $.063 E+00$ \\
\hline & & & & 694 & & & & & \\
\hline
\end{tabular}


Table 3. Photoneutron production from a 20-MeV beam following perturbations of the $(\gamma, n)$ and $(\gamma, 2 n)$ cross sections; the relative difference to the base case is also shown.

\begin{tabular}{|c|c|c|c|c|c|c|c|c|c|}
\hline$(\gamma, \mathrm{n})$ & $\delta_{(\gamma, 2 n)}$ & $f_{(\gamma, n)}$ & $f_{(\gamma, 2 n)}$ & $f_{(y, f)}$ & $<N_{r}>$ & $<N_{n}^{t}>$ & $<E_{n}^{t}>$ & $\left.<N_{n}^{d}\right\rangle$ & $<E_{n}^{d}>$ \\
\hline \multirow{10}{*}{0.00} & \multirow{2}{*}{0.00} & & & 0.2809 & 5.761E-03 & 1.126E-02 & $1.322 \mathrm{E}+00$ & $2.481 \mathrm{E}-05$ & $1.088 \mathrm{E}+00$ \\
\hline & & --- & --- & --- & --- & --- & --- & --- & --- \\
\hline & \multirow{2}{*}{-0.05} & 0.4889 & 0.2276 & 0.2835 & 5.694E-03 & 1.130E-02 & $1.327 \mathrm{E}+00$ & 2.486E-05 & $1.094 \mathrm{E}+00$ \\
\hline & & 0.0134 & -0.0385 & 0.0095 & -0.0115 & 0.0035 & 0.0042 & 0.0021 & 0.0057 \\
\hline & \multirow{2}{*}{-0.10} & 0.4960 & 0.2169 & 0.2871 & 5.662E-03 & 1.120E-02 & $1.332 \mathrm{E}+00$ & & $1.098 \mathrm{E}+00$ \\
\hline & & 0.0282 & -0.0838 & 0.0223 & -0.0171 & -0.0057 & 0.0078 & -0.0073 & 0.0096 \\
\hline & \multirow{2}{*}{-0.15} & 0.5004 & 0.2082 & 0.2914 & 5.532E-03 & 1.093E-02 & $1.335 \mathrm{E}+00$ & $2.401 \mathrm{E}-05$ & $1.098 \mathrm{E}+00$ \\
\hline & & 0.0374 & -0.1205 & 0.0374 & -0.0397 & -0.0295 & 0.0101 & -0.0324 & 0.0095 \\
\hline & \multirow{2}{*}{-0.20} & 0.5063 & 0.2002 & 0.2935 & $5.488 \mathrm{E}-03$ & 1.094E-02 & $1.349 \mathrm{E}+00$ & 2.415E-05 & $1.111 \mathrm{E}+00$ \\
\hline & & 0.0495 & -0.1542 & 0.0450 & -0.0473 & -0.0289 & 0.0207 & -0.0265 & 0.0218 \\
\hline \multirow{10}{*}{-0.05} & \multirow{2}{*}{0.00} & 4685 & 0.2427 & 2889 & $5.620 \mathrm{E}-03$ & 1.125E-02 & $1.321 \mathrm{E}+00$ & -05 & $1.091 \mathrm{E}+00$ \\
\hline & & -0.0289 & 0.0250 & 0.0285 & -0.0244 & -0.0011 & -0.0005 & -0.0053 & 0.0027 \\
\hline & \multirow{2}{*}{-0.05} & 0.4742 & 0.2335 & 0.2924 & 5.576E-03 & 1.120E-02 & $1.331 \mathrm{E}+00$ & $2.462 \mathrm{E}-05$ & $1.093 E+00$ \\
\hline & & -0.0171 & -0.0138 & 0.0410 & -0.0320 & -0.0058 & 0.0070 & -0.0078 & 0.0051 \\
\hline & \multirow{2}{*}{-0.10} & 0.4811 & 0.2230 & 0.2959 & 5.500 & 1.102E-02 & $1.336 \mathrm{E}+00$ & $2.428 \mathrm{E}-05$ & $1.099 E+00$ \\
\hline & & -0.0027 & -0.0582 & 0.0537 & -0.0452 & -0.0215 & 0.0110 & -0.0214 & \\
\hline & \multirow{2}{*}{-0.15} & 0.4879 & 0.2136 & 2985 & $5.450 \mathrm{E}-03$ & 1.094E-02 & $1.342 \mathrm{E}+00$ & 2.403E-05 & 1.105 \\
\hline & & 0.0114 & -0.0976 & 0.0626 & -0.0 & -0.0 & 0.01 & & 0.0157 \\
\hline & \multirow[b]{2}{*}{-0.20} & 0.4933 & & 3016 & 5.353 & 1.075E-02 & $1.351 \mathrm{E}+00$ & 2.370 & 1.11 \\
\hline & & 0.0225 & & 0739 & -0.0 & & 222 & & \\
\hline \multirow{10}{*}{-0.10} & \multirow{2}{*}{0.00} & 4561 & 0.2481 & 2957 & 5.47 & 1.109E-02 & 1.327 & $E-05$ & $1.093 E+00$ \\
\hline & & -0.0545 & 0.0482 & 0530 & -0.05 & & & & \\
\hline & \multirow{2}{*}{-0.05} & 0.4632 & 0.2393 & 0.2975 & $5.425 \mathrm{E}-03$ & 1.104E-02 & $1.334 \mathrm{E}+00$ & 2.424 & $1.098 \mathrm{E}+00$ \\
\hline & & -0.0398 & & 0.0592 & -0.0 & & & & 0.0098 \\
\hline & \multirow{2}{*}{.10} & 0.4668 & 0.2315 & 0.3017 & 5.365E-03 & 1.090E-02 & 1.337E+00 & 2.394E-05 & $1.100 \mathrm{E}+00$ \\
\hline & & -0.0324 & -0.0222 & .0744 & -0.0686 & -0.0328 & 0.0114 & -0.0352 & 0.0112 \\
\hline & \multirow{2}{*}{-0.15} & 0.4751 & 0.2193 & 0.3056 & $5.299 \mathrm{E}-03$ & 1.075E-02 & $1.346 \mathrm{E}+00$ & $2.366 \mathrm{E}-05$ & $1.106 \mathrm{E}+00$ \\
\hline & & -0.0151 & -0.0735 & 0880 & -0.0801 & & & -0.0462 & \\
\hline & \multirow{2}{*}{-0.20} & 0.4796 & 0.2084 & 3119 & 5.230 & 1.06 & $1.356 \mathrm{E}+00$ & 2.345E-05 & $1.115 \mathrm{E}+00$ \\
\hline & & & & & & & & & \\
\hline \multirow{10}{*}{-0.15} & & & & & 5.369E-03 & $1.106 \mathrm{E}-02$ & $1.331 \mathrm{E}+00$ & $2.431 \mathrm{E}-05$ & $1.098 \mathrm{E}+00$ \\
\hline & & & & & & & & & \\
\hline & & 0.4473 & & 3093 & 5.302E-03 & $1.0 \mathrm{~s}$ & $E+00$ & E-05 & $1.099 \mathrm{E}+00$ \\
\hline & -0.05 & & & & & & & & \\
\hline & -010 & & & & 5.23 & & 1.34 & & 1.10 \\
\hline & & & & & & & & & \\
\hline & & 0.4567 & 0.226 & 3167 & 5.152 & 1.065E-02 & 1.354 & 2.352E-05 & $1.112 \mathrm{E}+00$ \\
\hline & & & & & & & & & \\
\hline & & & & & 5.11 & $=-02$ & 1.36 & & 1.116 \\
\hline & & & & & & & & & \\
\hline & & 0.4289 & 0.261 & 3099 & $5.212 \mathrm{E}-03$ & 1.087E-02 & $1.334 \mathrm{E}+00$ & $2.381 \mathrm{E}-05$ & $1.100 \mathrm{E}+00$ \\
\hline & & -0.1108 & 0.1032 & 1034 & -0.0952 & -0.0352 & 0.0092 & -0.0402 & 0.0115 \\
\hline & & 0.4331 & 0.2534 & 0.3135 & 5.162E-03 & 1.078E-02 & $1.340 \mathrm{E}+00$ & 2.374E-05 & $1.103 \mathrm{E}+00$ \\
\hline & & & & & & & & & \\
\hline & & & 0.2406 & 3206 & 5.10 & 1.07 & 1.352 & $E-05$ & $1.111 \mathrm{E}+00$ \\
\hline-0.2 & & & & & & & & & \\
\hline & & 0.4417 & 0.2348 & 0.3236 & $5.017 \mathrm{E}-03$ & $1.045 \mathrm{E}-02$ & $1.352 \mathrm{E}+00$ & $2.298 \mathrm{E}-05$ & $1.112 \mathrm{E}+00$ \\
\hline & & & & & & -0.0720 & 0.0227 & -0.0736 & 0.0223 \\
\hline & & 0.4540 & 0.2201 & 0.3259 & 4.963E-03 & 1.04 & $1.370 \mathrm{E}+00$ & 2.303E-05 & $1.126 \mathrm{E}+00$ \\
\hline & & & & & & & & & 0.0 \\
\hline
\end{tabular}


Table 4. Relative errors (1- $\sigma)$ of photoneutron production from a 10-MeV beam following perturbations of the $(\gamma, \boldsymbol{n})$ and $(\gamma, 2 n)$ cross sections; mean values are listed in Table 1.

\begin{tabular}{|c|c|c|c|c|c|c|c|c|c|}
\hline$\delta_{(\gamma, \mathrm{n})}$ & $\delta_{(\gamma, 2 n)}$ & $f_{(y, n)}$ & $f_{(\gamma, 2 n)}$ & $f_{(y, f)}$ & $<N_{r}>$ & $<N_{n}^{t}>$ & $<E_{n}^{t}>$ & $<N_{n}^{d}>$ & $<E_{n}^{d}>$ \\
\hline & 0.00 & 0.0092 & --- & 0.0139 & 0.0086 & 0.0086 & 0.0007 & 0.0088 & 0.0116 \\
\hline \multirow{4}{*}{0.00} & -0.05 & 0.0092 & --- & 0.0139 & 0.0086 & 0.0086 & 0.0008 & 0.0089 & 0.0118 \\
\hline & -0.10 & 0.0091 & --- & 0.0138 & 0.0085 & 0.0085 & 0.0008 & 0.0088 & 0.0117 \\
\hline & -0.15 & 0.0092 & --- & 0.0138 & 0.0085 & 0.0085 & 0.0007 & 0.0088 & 0.0115 \\
\hline & -0.20 & 0.0091 & --- & 0.0137 & 0.0085 & 0.0085 & 0.0008 & 0.0088 & 0.0118 \\
\hline \multirow{5}{*}{-0.05} & 0.00 & 0.0094 & --- & 0.0139 & 0.0086 & 0.0086 & 0.0008 & 0.0089 & 0.0118 \\
\hline & -0.05 & 0.0093 & --- & 0.0140 & 0.0086 & 0.0086 & 0.0008 & 0.0089 & 0.0118 \\
\hline & -0.10 & 0.0093 & --- & 0.0138 & 0.0086 & 0.0086 & 0.0008 & 0.0089 & 0.0119 \\
\hline & -0.15 & 0.0094 & --- & 0.0139 & 0.0086 & 0.0086 & 0.0008 & 0.0089 & 0.0119 \\
\hline & -0.20 & 0.0094 & --- & 0.0138 & 0.0086 & 0.0086 & 0.0008 & 0.0089 & 0.0118 \\
\hline \multirow{5}{*}{-0.10} & 0.00 & 0.0096 & --- & 0.0139 & 0.0087 & 0.0087 & 0.0008 & 0.0090 & 0.0120 \\
\hline & -0.05 & 0.0096 & --- & 0.0139 & 0.0087 & 0.0087 & 0.0008 & 0.0090 & 0.0120 \\
\hline & -0.10 & 0.0096 & --- & 0.0139 & 0.0087 & 0.0087 & 0.0008 & 0.0090 & 0.0120 \\
\hline & -0.15 & 0.0096 & --- & 0.0138 & 0.0087 & 0.0087 & 0.0008 & 0.0090 & 0.0120 \\
\hline & -0.20 & 0.0096 & --- & 0.0138 & 0.0087 & 0.0087 & 0.0008 & 0.0090 & 0.0120 \\
\hline \multirow{5}{*}{-0.15} & 0.00 & 0.00 & --- & 0.0140 & 0.00 & 0.0088 & 0.0008 & 0.0091 & 0.0122 \\
\hline & -0.05 & & --- & 0.01 & & & & & 0.0122 \\
\hline & -0.10 & 0.0099 & --- & 0.0141 & 0.0088 & 0.0088 & 0.0008 & 0.0092 & 0.0124 \\
\hline & -0.15 & & --- & & & & 0.0 & 091 & 0.0123 \\
\hline & -0.20 & & --- & & & & & & 0.0123 \\
\hline \multirow{5}{*}{-0.20} & 0.00 & 0.0101 & --- & 0.0140 & 0.0089 & 0.0089 & 0.0008 & 0.0092 & 0.0125 \\
\hline & -0.05 & & --- & & & & & & 0.0125 \\
\hline & -0.10 & 0.0101 & --- & 0.01 & 0.0089 & 0.0 & 0.0008 & 0.0092 & 0.0123 \\
\hline & -0.15 & 0.0101 & --- & 0.0139 & & 0.0089 & & 0.0092 & 0.0123 \\
\hline & -0.20 & 0.0100 & --- & 0.0141 & 0.0089 & 0.0089 & 0.0008 & 0.0092 & 0.0124 \\
\hline
\end{tabular}

Table 5. Relative errors (1- $\sigma)$ of photoneutron production from a 15-MeV beam following perturbations of the $(\gamma, n)$ and $(\gamma, 2 n)$ cross sections; mean values are listed in Table 2.

\begin{tabular}{|c|c|c|c|c|c|c|c|c|c|}
\hline$\delta_{(\gamma, \mathrm{n})}$ & $\delta_{(\gamma, 2 n)}$ & $f_{(\gamma, n)}$ & $f_{(\gamma, 2 n)}$ & $f_{(\gamma, f)}$ & $<N_{r}>$ & $<N_{n}^{t}>$ & $<E_{n}^{t}>$ & $<N_{n}^{d}>$ & $<E_{n}^{d}>$ \\
\hline & 0.00 & 0.0057 & 0.0104 & 0.0080 & 0.0050 & 0.0050 & 0.0008 & 0.0054 & 0.0114 \\
\hline \multirow{4}{*}{0.00} & -0.05 & 0.0058 & 0.0106 & 0.0080 & 0.0050 & 0.0050 & 0.0008 & 0.0055 & 0.0113 \\
\hline & -0.10 & 0.0057 & 0.0108 & 0.0080 & 0.0050 & 0.0050 & 0.0008 & 0.0055 & 0.0114 \\
\hline & -0.15 & 0.0058 & 0.0112 & 0.0080 & 0.0050 & 0.0050 & 0.0008 & 0.0055 & 0.0116 \\
\hline & -0.20 & 0.0058 & 0.0115 & 0.0080 & 0.0051 & 0.0051 & 0.0008 & 0.0055 & 0.0116 \\
\hline \multirow{5}{*}{-0.05} & 0.00 & 0.0059 & 0.0104 & 0.0080 & 0.0051 & 0.0051 & 0.0008 & 0.0055 & 0.0114 \\
\hline & -0.05 & 0.0059 & 0.0106 & 0.0080 & 0.0051 & 0.0051 & 0.0008 & 0.0055 & 0.0116 \\
\hline & -0.10 & 0.0059 & 0.0109 & 0.0080 & 0.0051 & 0.0051 & 0.0008 & 0.0055 & 0.0116 \\
\hline & -0.15 & 0.0059 & 0.0111 & 0.0080 & 0.0051 & 0.0051 & 0.0008 & 0.0055 & 0.0116 \\
\hline & -0.20 & 0.0059 & 0.0115 & 0.0081 & 0.0051 & 0.0051 & 0.0008 & 0.0055 & 0.0116 \\
\hline \multirow{5}{*}{-0.10} & 0.00 & 0.0060 & 0.0103 & 0.0081 & 0.0051 & 0.0051 & 0.0008 & 0.0055 & 0.0116 \\
\hline & -0.05 & 0.0060 & 0.0107 & 0.0080 & 0.0051 & 0.0051 & 0.0008 & 0.0056 & 0.0116 \\
\hline & -0.10 & 0.0060 & 0.0108 & 0.0081 & 0.0051 & 0.0051 & 0.0008 & 0.0055 & 0.0116 \\
\hline & -0.15 & 0.0060 & 0.0111 & 0.0080 & 0.0051 & 0.0051 & 0.0008 & 0.0056 & 0.0117 \\
\hline & -0.20 & 0.0060 & 0.0114 & 0.0080 & 0.0051 & 0.0051 & 0.0008 & 0.0056 & 0.0118 \\
\hline \multirow{5}{*}{-0.15} & 0.00 & 0.0061 & 0.0104 & 0.0081 & 0.0051 & 0.0051 & 0.0008 & 0.0056 & 0.0116 \\
\hline & -0.05 & 0.0061 & 0.0106 & 0.0081 & 0.0052 & 0.0052 & 0.0008 & 0.0056 & 0.0117 \\
\hline & -0.10 & 0.0062 & 0.0109 & 0.0081 & 0.0052 & 0.0052 & 0.0008 & 0.0056 & 0.0118 \\
\hline & -0.15 & 0.0062 & 0.0112 & 0.0080 & 0.0052 & 0.0052 & 0.0008 & 0.0056 & 0.0118 \\
\hline & -0.20 & 0.0062 & 0.0115 & 0.0081 & 0.0052 & 0.0052 & 0.0008 & 0.0056 & 0.0119 \\
\hline \multirow{5}{*}{-0.20} & 0.00 & 0.0063 & 0.0104 & 0.0081 & 0.0052 & 0.0052 & 0.0008 & 0.0056 & 0.0118 \\
\hline & -0.05 & 0.0063 & 0.0106 & 0.0081 & 0.0052 & 0.0052 & 0.0008 & 0.0056 & 0.0118 \\
\hline & -0.10 & 0.0063 & 0.0109 & 0.0081 & 0.0052 & 0.0052 & 0.0008 & 0.0057 & 0.0118 \\
\hline & -0.15 & & 0.0111 & & 0.0052 & 0.0052 & 0.0008 & 0.0057 & 0.0119 \\
\hline & -0.20 & 0.0063 & 0.0115 & 0.0081 & 0.0052 & 0.0052 & 0.0008 & 0.0057 & 0.0121 \\
\hline
\end{tabular}


Table 6. Relative errors (1- $\sigma)$ of photoneutron production from a 20-MeV beam following perturbations of the $(\gamma, n)$ and $(\gamma, 2 n)$ cross sections; mean values are listed in Table 3 .

\begin{tabular}{|c|c|c|c|c|c|c|c|c|c|}
\hline$\delta_{(\gamma, \mathrm{n})}$ & $\delta_{(\gamma, 2 n)}$ & $f_{(\gamma, n)}$ & $f_{(\gamma, 2 n)}$ & $f_{(\gamma, f)}$ & $<N_{r}>$ & $<N_{n}^{t}>$ & $<E_{n}^{t}>$ & $<N_{n}^{d}>$ & $<E_{n}^{d}>$ \\
\hline \multirow{5}{*}{0.00} & 0.00 & 0.0053 & 0.0069 & 0.0065 & 0.0043 & 0.0043 & 0.0006 & 0.0046 & 0.0093 \\
\hline & -0.05 & 0.0053 & 0.0071 & 0.0065 & 0.0043 & 0.0043 & 0.0006 & 0.0046 & 0.0093 \\
\hline & -0.10 & 0.0053 & 0.0073 & 0.0065 & 0.0043 & 0.0043 & 0.0006 & 0.0046 & 0.0093 \\
\hline & -0.15 & 0.0054 & 0.0075 & 0.0065 & 0.0043 & 0.0043 & 0.0006 & 0.0047 & 0.0094 \\
\hline & -0.20 & 0.0054 & 0.0076 & 0.0065 & 0.0043 & 0.0043 & 0.0006 & 0.0047 & 0.0094 \\
\hline \multirow{5}{*}{-0.05} & 0.00 & 0.0054 & 0.0070 & 0.0065 & 0.0043 & 0.0043 & 0.0006 & 0.0046 & 0.0093 \\
\hline & -0.05 & 0.0054 & 0.0071 & 0.0065 & 0.0043 & 0.0043 & 0.0006 & 0.0047 & 0.0093 \\
\hline & -0.10 & 0.0055 & 0.0073 & 0.0065 & 0.0043 & 0.0043 & 0.0006 & 0.0047 & 0.0093 \\
\hline & -0.15 & 0.0055 & 0.0074 & 0.0065 & 0.0044 & 0.0044 & 0.0006 & 0.0047 & 0.0095 \\
\hline & -0.20 & 0.0055 & 0.0076 & 0.0065 & 0.0044 & 0.0044 & 0.0006 & 0.0047 & 0.0096 \\
\hline \multirow{5}{*}{-0.10} & 0.00 & 0.0056 & 0.0070 & 0.0 & & & & & \\
\hline & -0.05 & 0.0056 & 0.0071 & 0.0065 & 0.0044 & 0.0044 & 0.0006 & 0.0047 & 0.0094 \\
\hline & -0.10 & 0.00 & & 0.0 & & & 0.0006 & 047 & 0.0095 \\
\hline & -0.15 & 0.0056 & 0.0075 & 0.0065 & 0.0044 & 0.0044 & 0.0006 & 0.0047 & 0.0096 \\
\hline & -0.20 & 0.0056 & 0.0077 & 0.0065 & 0.0044 & 0.0044 & 0.0006 & 0.0047 & 0.0096 \\
\hline \multirow{5}{*}{-0.15} & 0.00 & 0.0057 & 0.0070 & 0.0065 & 0.0044 & 0.0044 & 0.0006 & 0.0047 & 0.0094 \\
\hline & -0.05 & 0.0057 & 0.0072 & 0.0065 & 0.0044 & 0.0044 & 0.0006 & 0.0047 & 0.0094 \\
\hline & -0.10 & 0.0057 & 0.0073 & 0.0066 & 0.0044 & 0.0044 & 0.0006 & 0.0047 & 0.0094 \\
\hline & -0.15 & 0.0057 & 0.0075 & 0.0065 & 0.0044 & 0.0044 & 0.0006 & 0.0047 & 0.0096 \\
\hline & -0.20 & 0.0057 & 0.0077 & 0.0066 & 0.0044 & 0.0044 & 0.0006 & 0.0047 & 0.0096 \\
\hline \multirow{5}{*}{-0.20} & 0.00 & 0.0058 & 0.0070 & 0.0066 & 0.0044 & 0.0044 & 0.0006 & 0.0047 & 0.0095 \\
\hline & -0.05 & 0.0058 & 0.0071 & 0.0066 & 0.0044 & 0.0044 & 0.0006 & 0.0047 & 0.0096 \\
\hline & -0.10 & 0.0058 & 0.0073 & 0.0065 & 0.0044 & 0.0044 & 0.0006 & 0.0047 & 0.0096 \\
\hline & -0.15 & 0.0059 & 0.0075 & 0.0066 & 0.0045 & 0.0045 & 0.0006 & 0.0048 & 0.0096 \\
\hline & -0.20 & 0.0059 & 0.0077 & 0.0066 & 0.0045 & 0.0045 & 0.0006 & 0.0048 & 0.0097 \\
\hline
\end{tabular}

The results show that the fraction of $(\gamma, 2 n)$ and $(\gamma, f)$ reactions increases for each $(\gamma, n)$ perturbation: this is a direct result of the $(\gamma, n)$ cross section decrease. Fig. 4 shows the normalized reaction cross sections to illustrate this. As expected, the total number of reactions decreases as a result of the decrease in total cross section.

The number of neutrons crossing the face of the detector is less than the number of neutrons emitted from the target by a constant factor equal to the solid angle subtended by the detector face. The average energy of the neutrons crossing the detector face is reduced from those emitted from the target by a constant factor proportional to the attenuation in the lead shielding. For the $20-\mathrm{MeV}$ extreme case, a perturbation of $-20 \%$ to both cross sections, the average number of reactions is reduced by about $14 \%$ which results in only a $7 \%$ decrease in the number of neutrons crossing the detector face. The average number of neutrons emitted per reaction, however, increases. This is expected because $(\gamma, 2 n)$ and $(\gamma, f)$ reactions occur instead of $(\gamma, n)$ reactions. The larger number of fission neutrons results in only a slight increase, approximately $3.5 \%$, in the average neutron energy.

For the $10-\mathrm{MeV}$ case, the entire photon spectrum is below the threshold of a $(\gamma, 2 n)$ reaction $\left(E_{\mathrm{th}}=11.28 \mathrm{MeV}\right)$, therefore, perturbations to the $(\gamma, 2 n)$ cross section should have no effect on the results. However, Table 1 shows some slight variation in the observable quantities. These fluctuations are outside of the 1- $\sigma$ errors listed in Table 4 and can be attributed to statistical uncertainty in the Monte Carlo calculations. 
Fig. 5 through Fig. 10 shows the results from Tables 1 through 3 in graphical form. In general, the percentage of change in photoneutron production rate decreases with the decrease in the cross-sections while the percentage of change in the average photoneutron energy increases with the decrease in the cross sections. This trend is present for all three endpoint energies.

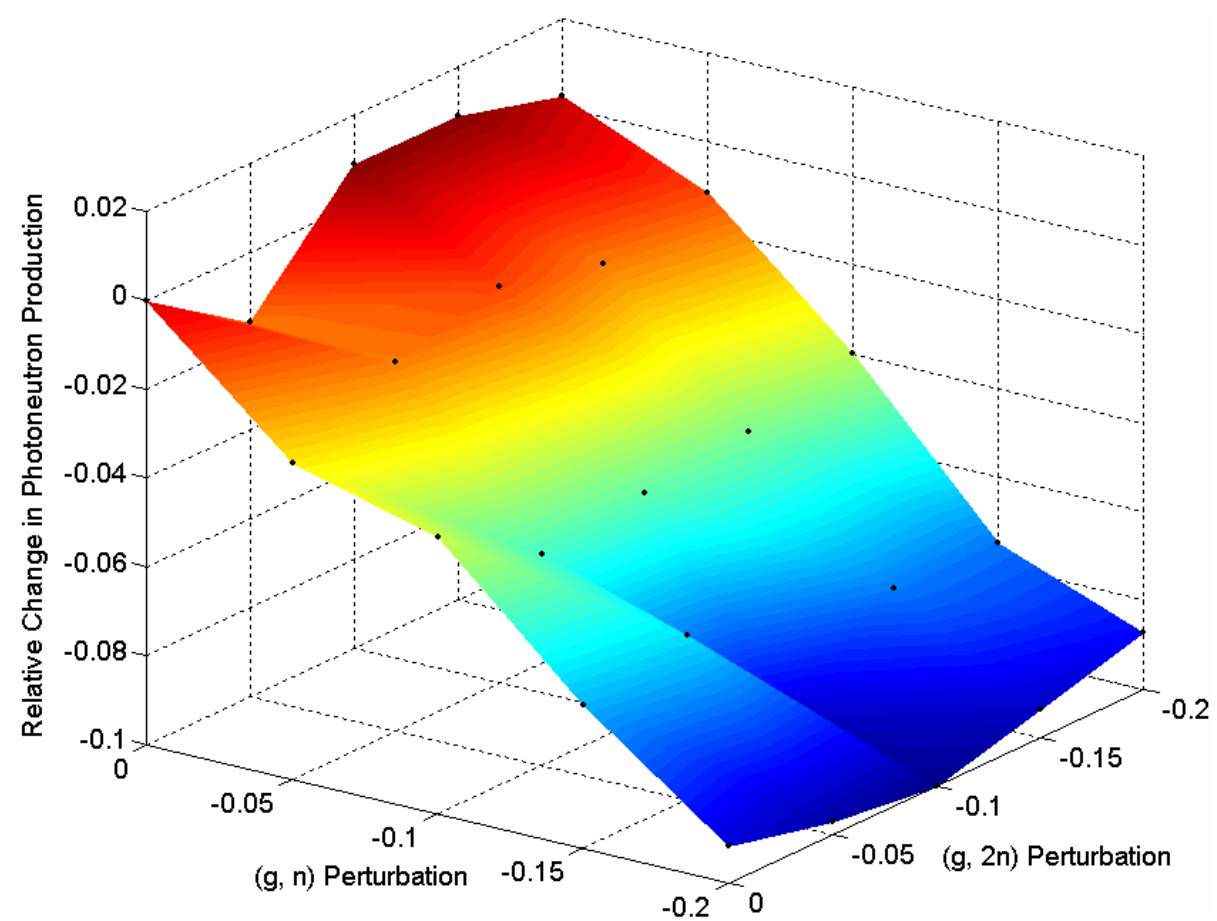

Fig. 5. Change in photoneutron production, $<N_{n}^{t}>$, from a $10-\mathrm{MeV}$ beam following perturbations in the $(\gamma, n)$ and $(\gamma, 2 n)$ cross sections. 


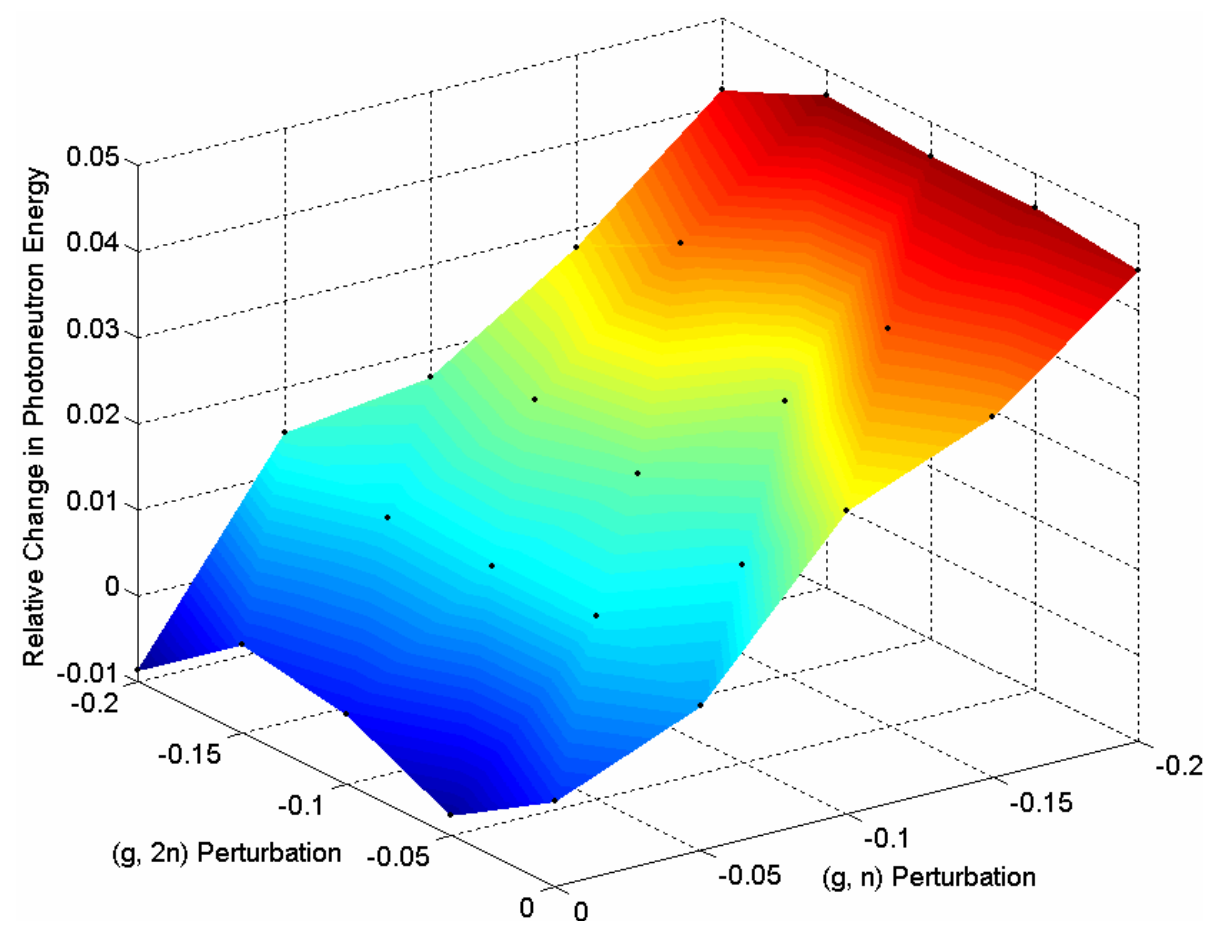

Fig. 6. Change in average photoneutron energy, $\left\langle E_{n}^{t}\right\rangle$, from a $10-\mathrm{MeV}$ beam following perturbations in the $(\gamma, n)$ and $(\gamma, 2 n)$ cross sections.

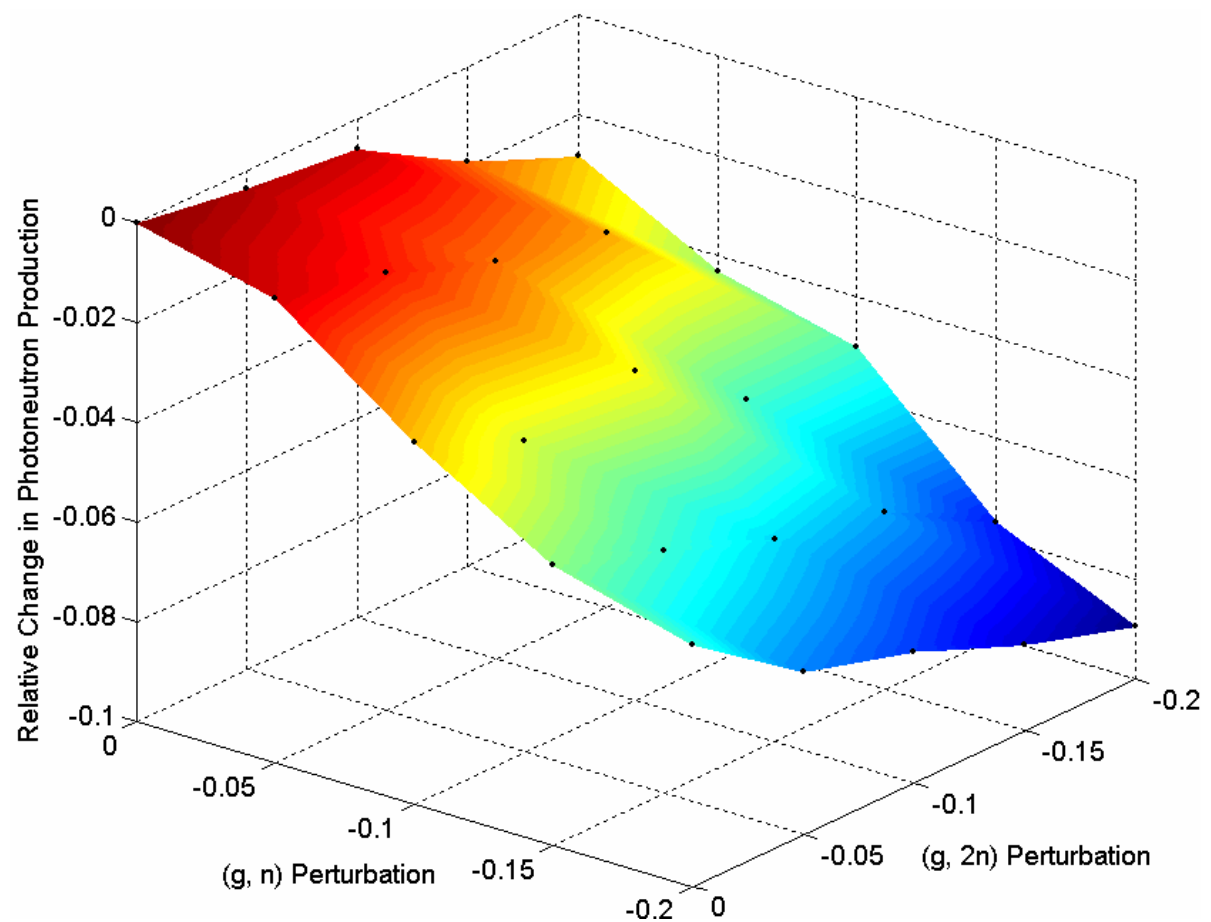

Fig. 7. Change in photoneutron production, $<N_{n}^{t}>$, from a 15-MeV beam following perturbations in the $(\gamma, n)$ and $(\gamma, 2 n)$ cross sections. 


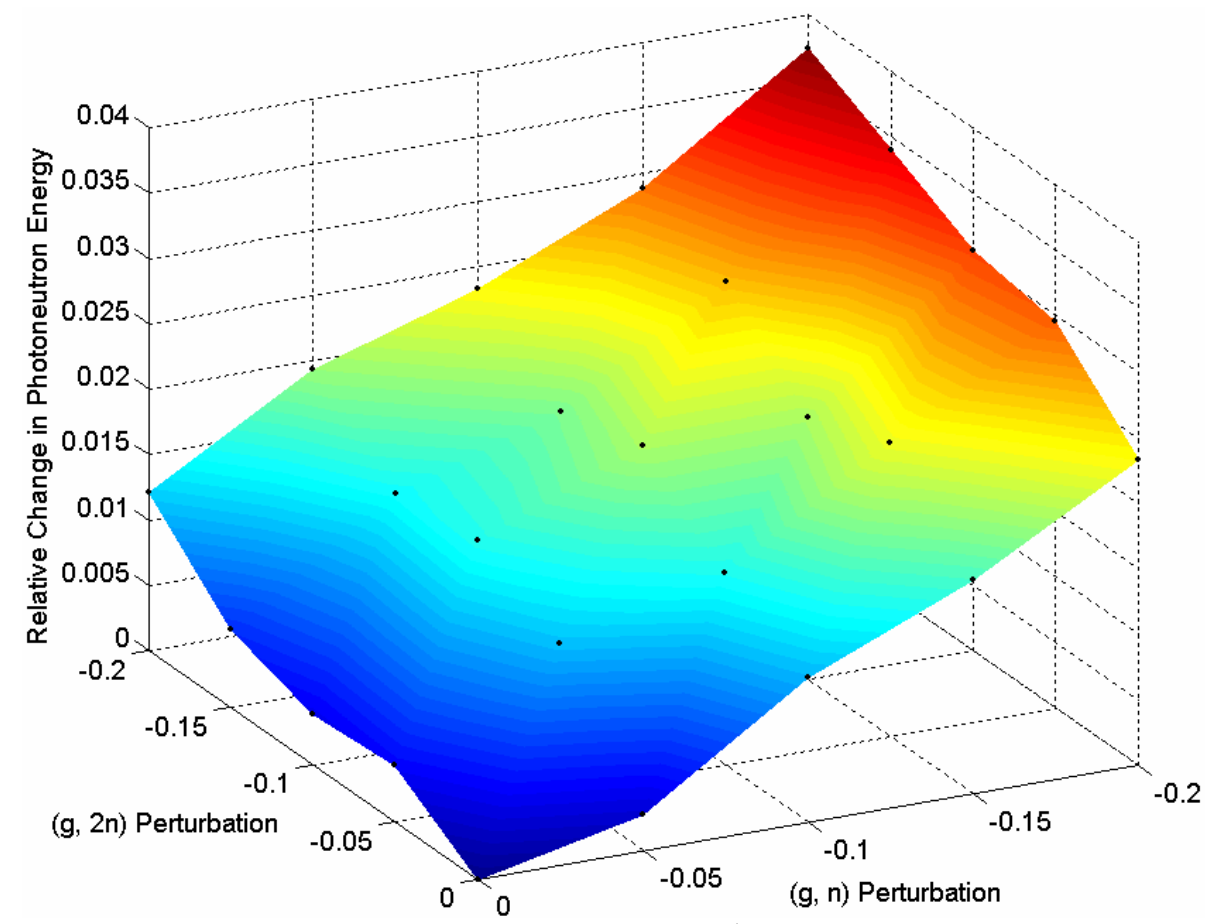

Fig. 8. Change in average photoneutron energy, $\left\langle E_{n}^{t}\right\rangle$, from a 15-MeV beam following perturbations in the $(\gamma, n)$ and $(\gamma, 2 n)$ cross sections.

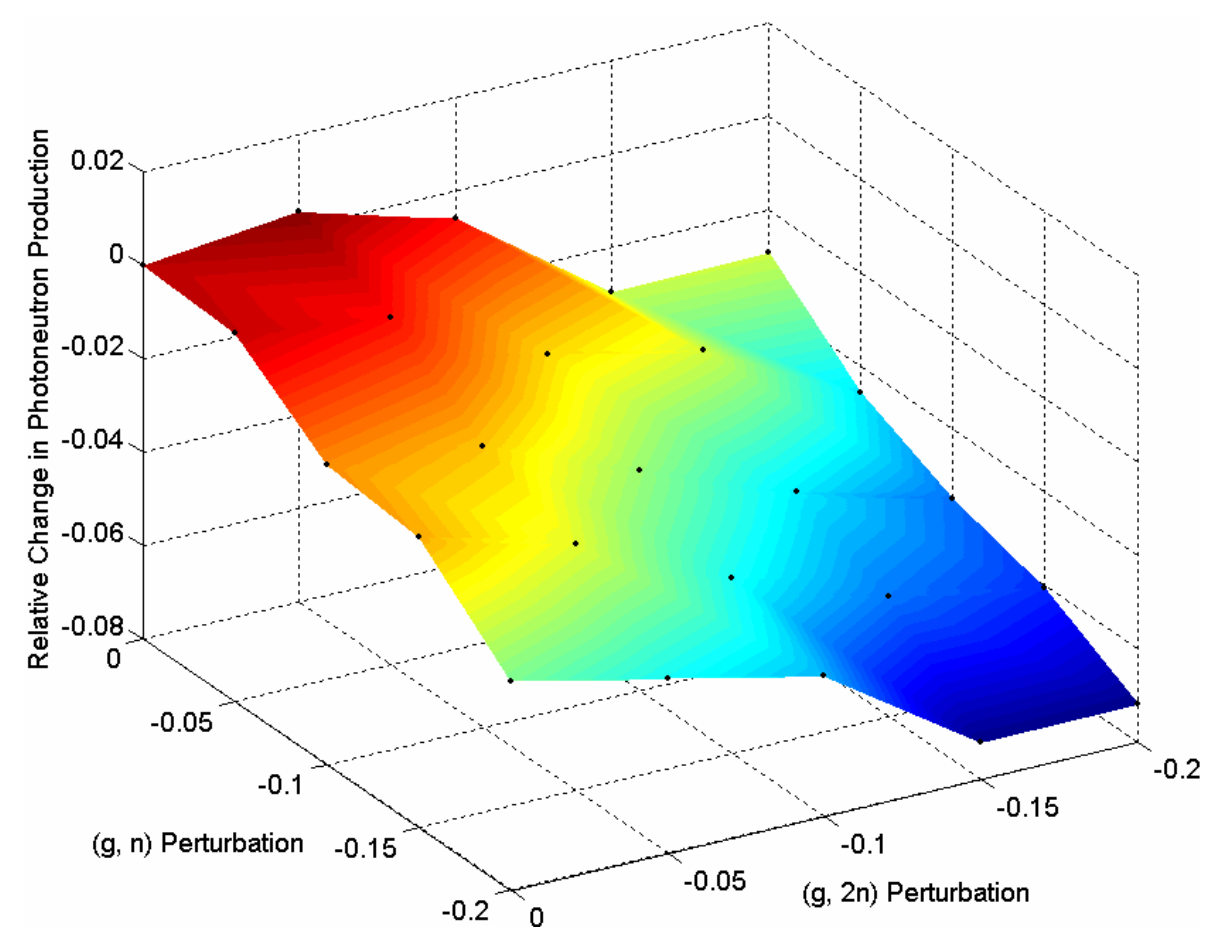

Fig. 9. Change in photoneutron production, $\left\langle N_{n}^{t}\right\rangle$, from a 20-MeV beam following perturbations in the $(\gamma, n)$ and $(\gamma, 2 n)$ cross sections. 


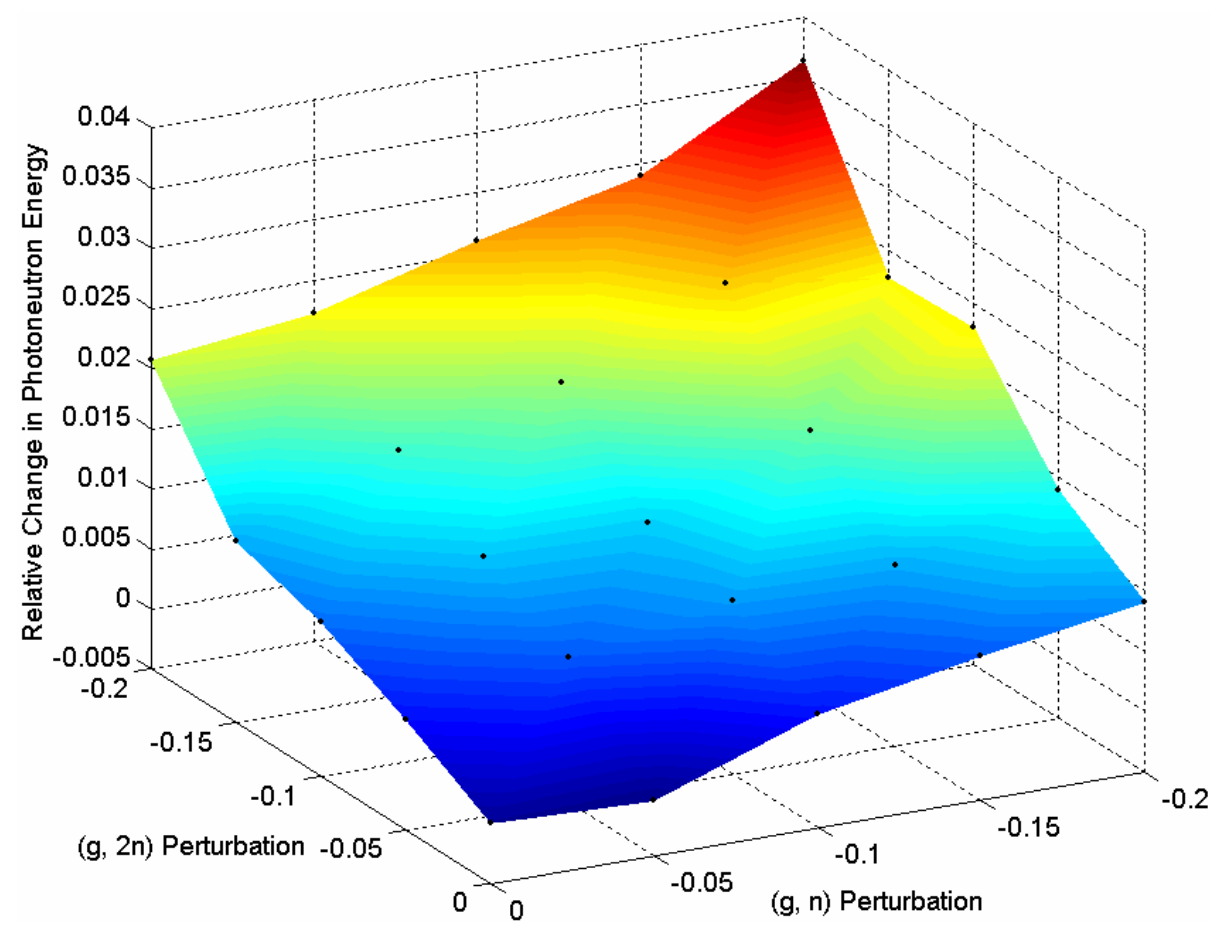

Fig. 10. Change in average photoneutron energy, $\left\langle E_{n}^{t}\right\rangle$, from a $20-\mathrm{MeV}$ beam following perturbations in the $(\gamma, n)$ and $(\gamma, 2 n)$ cross sections.

The photoneutron production sensitivity may be computed by dividing the change in the number of neutrons produced by the magnitude of the cross-section perturbation. For simplicity, the photoneutron production sensitivity is computed with respect to individual changes in the $(\gamma, n)$ and $(\gamma, 2 n)$ cross sections:

$$
\begin{gathered}
S_{1}=\left.\frac{\delta_{N_{n}^{t}}}{\delta_{(\gamma, n)}}\right|_{\delta_{(\gamma, 2 n)}=0}, \\
S_{2}=\left.\frac{\delta_{N_{n}^{t}}}{\delta_{(\gamma, 2 n)}}\right|_{\delta_{(\gamma, n)}=0} .
\end{gathered}
$$

The sensitivities $S_{1}$ and $S_{2}$ were computed for each cross-section perturbation for each beam energy. Table 7 lists the $S_{1}$ values and Table 8 lists the $S_{2}$ values. The1- $\sigma$ relative error was also computed for each sensitivity value. The $S_{1}$ value is greater than the $S_{2}$ value for all beam energies, i.e., the neutron production is more sensitive to changes the $(\gamma, n)$ cross section than to changes in the $(\gamma, 2 n)$ cross section. Also, for smaller perturbations $(-0.05$ and -0.10$)$, both sensitivity values are generally inside of $1-\sigma$. For larger perturbations, the sensitivity lies outside of $1-\sigma$. As expected, the $10-\mathrm{MeV}$ beam is largely insensitive to changes in the $(\gamma, 2 n)$ cross section. 
Table 7. Photoneutron production sensitivity with respect to perturbations in the $(\gamma, n)$ reaction cross section $\left(S_{1}\right)$; the $1-\sigma$ relative error is also shown.

\begin{tabular}{|c|c|c|c|}
\hline \multirow{2}{*}{$\delta_{(\gamma, n)}$} & \multicolumn{3}{|c|}{ Beam Energy [MeV] } \\
\cline { 2 - 4 } & 10 & 15 & 20 \\
\hline \hline \multirow{2}{*}{0.05} & 0.5806 & 0.1383 & 0.0219 \\
& 0.1728 & 0.1012 & 0.0865 \\
\hline \multirow{2}{*}{0.10} & 0.3791 & 0.2740 & 0.1569 \\
& 0.0871 & 0.0510 & 0.0435 \\
\hline \multirow{2}{*}{0.15} & 0.4530 & 0.2921 & 0.1194 \\
& 0.0587 & 0.0343 & 0.0292 \\
\hline \multirow{2}{*}{0.20} & 0.4591 & 0.2579 & 0.1759 \\
& 0.0445 & 0.0259 & 0.0220 \\
\hline
\end{tabular}

Table 8. Photoneutron production sensitivity with respect to perturbations in the $(\gamma, 2 n)$ reaction cross section $\left(S_{2}\right)$; the $1-\sigma$ relative error is also shown.

\begin{tabular}{||c||c|c|c||}
\hline \multirow{2}{*}{$\delta_{(\gamma, 2 n)}$} & \multicolumn{3}{c|}{ Beam Energy [MeV] } \\
\cline { 2 - 4 }-0.05 & 0.3156 & 0.0722 & -0.0701 \\
& 0.1714 & 0.1007 & 0.0862 \\
\hline \multirow{2}{*}{-0.10} & -0.0895 & 0.0586 & 0.0570 \\
& 0.0853 & 0.0503 & 0.0432 \\
\hline \multirow{2}{*}{-0.15} & -0.0597 & 0.1259 & 0.1964 \\
& 0.0569 & 0.0336 & 0.0289 \\
\hline \multirow{2}{*}{-0.20} & -0.0132 & 0.1405 & 0.1444 \\
& 0.0426 & 0.0253 & 0.0217 \\
\hline
\end{tabular}

\subsection{Detector Response Results}

The MCNP-PoliMi detection post-processor was used to predict the time-of-flight spectrum recorded by the $50-$ by $-50-\mathrm{cm}^{2}$ liquid scintillation detector. The post-processing code uses the energy deposited in each particle collision (recorded by MCNP-PoliMi) and converts it into light produced by the scintillator. The total light generated by each particle track is computed and compared to a user-defined detection threshold. If the total light created during the pulse generation time is greater than the threshold, one count is recorded at a specific time. The time difference between consecutive counts is compared to the deadtime defined by the user. A detection threshold of $0.0793 \mathrm{MeVee}$ was applied in these simulations (this corresponds to the amount of light generated by a $500-\mathrm{keV}$ neutron). The deadtime was set to $80 \mathrm{~ns}$.

Fig. 11 shows the simulated time-of-flight count distribution from the $10-\mathrm{MeV}$ beam using the base-case cross sections. The small, initial peak (at times less than $20 \mathrm{~ns}$ ) is generated by the arrival of gamma rays from photonuclear interactions in the target. This peak is relatively narrow since all gamma rays travel at the same speed, and therefore arrive within a very short time window. The photoneutrons begin arriving at the detector at approximately $40 \mathrm{~ns}$. The width of the neutron peak is much greater than the gammaray peak because the neutrons have a distribution of velocities. The peak is shifted 
slightly to later times due to the large percentage of $(\gamma, n)$ reactions that occur from the $10-\mathrm{MeV}$ beam; on average, these neutrons are much slower than fission neutrons.

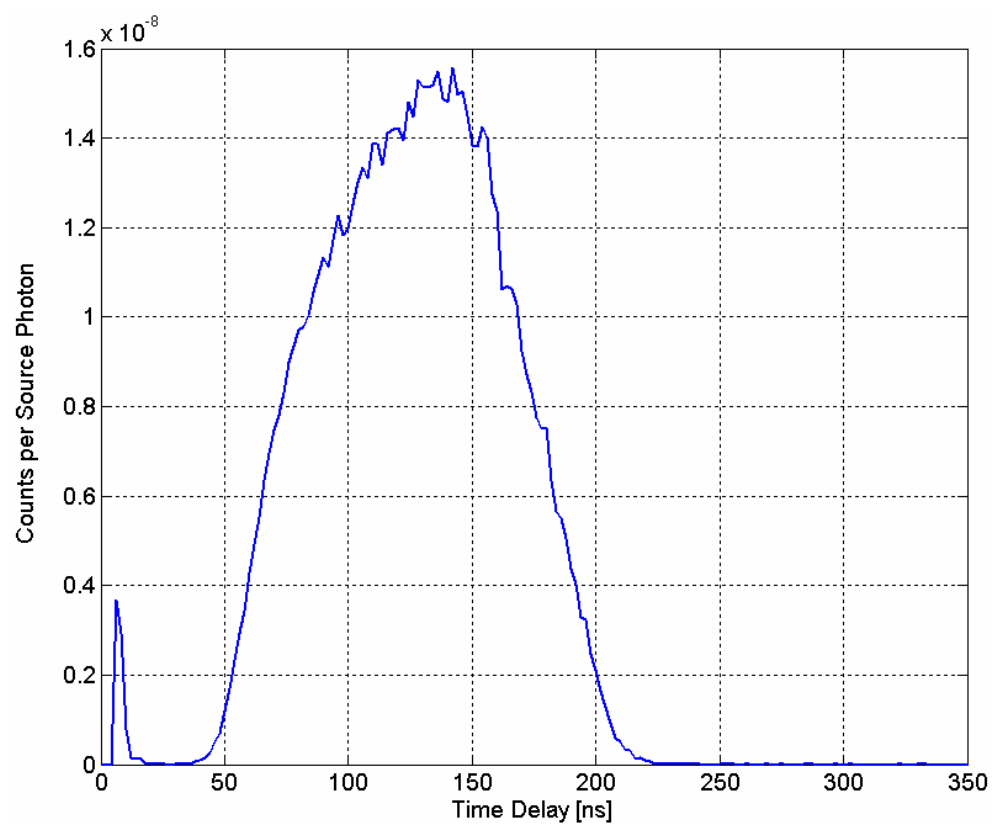

Fig. 11. Simulated time-of-flight spectrum generated with a $10-\mathrm{MeV}$ beam and a 0.0793-MeVee detection threshold.

In order to make a bulk comparison of each perturbation case, the neutron counts were integrated from 25 to $250 \mathrm{~ns}$. This integration range was held constant for all perturbation cases and for all beam energies.

Tables 9 through 11 list these results for the 10, 15 and 20-MeV beams. The trends are similar to those observed in the MCNP-PoliMi tally results discussed in the previous section. The detector response includes the effects of number of neutrons (deadtime) and their average energy (detection threshold). For this reason, the detector response is slightly less sensitive to the cross section perturbations than the bulk neutron production.

For example, consider the $15-\mathrm{MeV}$ beam case with -0.20 perturbations to both the $(\gamma, n)$ and $(\gamma, 2 n)$ cross sections. Table 2 shows the number of neutrons crossing the detector case decreases by approximately $9 \%$ following this perturbation; according to Table 10 , the number of counts decreases by only $5 \%$. This discrepancy can be explained by the energy of the neutrons: even though the total number of neutrons has decreased, their average energy - and therefore detection probability - has increased. This trend is observed in all cases. 
Table 9. MCNP-PoliMi time-integrated neutron counts from a 10-MeV beam; relative difference to the base case is also shown.

\begin{tabular}{c|c|c|c|c|c|}
\multicolumn{1}{c}{$\delta_{(\gamma, 2 n)}$} & 0.00 & -0.05 & -0.10 & -0.15 & -0.20 \\
\hline \multirow{2}{*}{0.00} & $1.5403 \mathrm{E}-06$ & $1.5040 \mathrm{E}-06$ & $1.4941 \mathrm{E}-06$ & $1.4544 \mathrm{E}-06$ & $1.3908 \mathrm{E}-06$ \\
& --- & -0.0235 & -0.0300 & -0.0558 & -0.0970 \\
\hline \multirow{2}{*}{0.05} & $1.4902 \mathrm{E}-06$ & $1.4924 \mathrm{E}-06$ & $1.4579 \mathrm{E}-06$ & $1.4686 \mathrm{E}-06$ & $1.4113 \mathrm{E}-06$ \\
& -0.0325 & -0.0311 & -0.0535 & -0.0465 & -0.0837 \\
\hline \multirow{2}{*}{0.10} & $1.5050 \mathrm{E}-06$ & $1.4988 \mathrm{E}-06$ & $1.4644 \mathrm{E}-06$ & $1.3854 \mathrm{E}-06$ & $1.4238 \mathrm{E}-06$ \\
& -0.0229 & -0.0269 & -0.0492 & -0.1006 & -0.0756 \\
\hline \multirow{2}{*}{0.15} & $1.5468 \mathrm{E}-06$ & $1.4867 \mathrm{E}-06$ & $1.4741 \mathrm{E}-06$ & $1.4335 \mathrm{E}-06$ & $1.4317 \mathrm{E}-06$ \\
& 0.0042 & -0.0348 & -0.0430 & -0.0693 & -0.0705 \\
\hline \multirow{2}{*}{0.20} & $1.4781 \mathrm{E}-06$ & $1.5219 \mathrm{E}-06$ & $1.4740 \mathrm{E}-06$ & $1.4154 \mathrm{E}-06$ & $1.4090 \mathrm{E}-06$ \\
& -0.0404 & -0.0119 & -0.0431 & -0.0811 & -0.0852 \\
\hline
\end{tabular}

Table 10. MCNP-PoliMi time-integrated neutron counts from a 15-MeV beam; relative difference to the base case is also shown.

\begin{tabular}{|c|c|c|c|c|c|}
\hline \multirow[b]{2}{*}{$\delta_{(\gamma, 2 n)}$} & \multicolumn{5}{|c|}{$\delta_{(\gamma, n)}$} \\
\hline & 0.00 & -0.05 & -0.10 & -0.15 & -0.20 \\
\hline \multirow{2}{*}{0.00} & $1.8411 \mathrm{E}-06$ & 1.8133E-06 & $1.7901 \mathrm{E}-06$ & $1.7670 \mathrm{E}-06$ & 1.7681E-06 \\
\hline & --- & -0.0151 & -0.0277 & -0.0403 & -0.0397 \\
\hline \multirow{2}{*}{-0.05} & $1.8366 \mathrm{E}-06$ & $1.7958 \mathrm{E}-06$ & 1.7767E-06 & 1.7806E-06 & 1.7503E-06 \\
\hline & -0.0024 & -0.0246 & -0.0350 & -0.0329 & -0.0493 \\
\hline \multirow{2}{*}{-0.10} & 1.8176E-06 & 1.7956E-06 & $1.8066 \mathrm{E}-06$ & 1.7567 & 1.7503 \\
\hline & -0.0128 & -0.0247 & -0.0187 & -0.0459 & -0.0493 \\
\hline \multirow{2}{*}{-0.15} & $1.8008 \mathrm{E}-06$ & $1.7995 \mathrm{E}-06$ & 1.7801E-06 & $1.7460 \mathrm{E}-06$ & 1.7306E-06 \\
\hline & -0.0219 & -0.0226 & -0.0331 & -0.0516 & -0.0601 \\
\hline \multirow{2}{*}{-0.20} & 1.7887E-06 & $1.7795 \mathrm{E}-06$ & $1.7544 \mathrm{E}-06$ & $1.7467 \mathrm{E}-06$ & $1.6982 \mathrm{E}-06$ \\
\hline & -0.0285 & -0.0335 & -0.0471 & -0.0513 & -0.0776 \\
\hline
\end{tabular}

Table 11. MCNP-PoliMi time-integrated neutron counts from a 20-MeV beam; relative difference to the base case is also shown.

\begin{tabular}{|c|c|c|c|c|c|}
\hline \multirow{2}{*}{$\delta_{(\gamma, 2 n)}$} & \multicolumn{5}{|c|}{$\delta_{(\gamma, n)}$} \\
\hline & 0.00 & -0.05 & -0.10 & -0.15 & -0.20 \\
\hline \multirow{2}{*}{0.00} & 2.9248E-06 & 2.9242E-06 & 2.8694E-06 & 2.8956E-06 & 2.8510E-06 \\
\hline & --- & -0.0002 & -0.0189 & -0.0100 & -0.0252 \\
\hline \multirow{2}{*}{-0.05} & $2.9428 \mathrm{E}-06$ & $2.8998 \mathrm{E}-06$ & 2.8704E-06 & $2.8716 \mathrm{E}-06$ & 2.8210E-06 \\
\hline & 0.0062 & -0.0085 & -0.0186 & -0.0182 & -0.0355 \\
\hline \multirow{2}{*}{-0.10} & 2.9172E-06 & $2.9406 \mathrm{E}-06$ & 2.8300E-06 & $2.9010 \mathrm{E}-06$ & 2.8142E-06 \\
\hline & -0.0026 & 0.0054 & -0.0324 & & -0.0378 \\
\hline \multirow{2}{*}{-0.15} & $2.8662 \mathrm{E}-06$ & 2.8322E-06 & $2.8380 \mathrm{E}-06$ & $2.8362 \mathrm{E}-06$ & $2.7642 \mathrm{E}-06$ \\
\hline & -0.0200 & -0.0317 & -0.0297 & -0.0 & -0.0549 \\
\hline \multirow{2}{*}{-0.20} & 2.9012E-06 & 2.8496E-06 & 2.8000E-06 & 2.7998E-06 & 2.8060E-06 \\
\hline & -0.0081 & -0.0257 & -0.0427 & -0.0427 & -0.0406 \\
\hline
\end{tabular}




\section{DISCUSSION AND CONCLUSIONS}

The sensitivity of the photoneutron production in DU to perturbations in the $(\gamma, n)$ and $(\gamma, 2 n)$ cross sections has been investigated using the MCNPX/MCNP-PoliMi code system. A new methodology has been developed that utilizes the modular nature of this code system to overcome the fact that MCNPX does have available sensitivity routines for photonuclear reactions. Thus an external calculation was performed for each crosssection perturbation to determine the change in the photonuclear source and MCNPPoliMi was used to propagate this perturbed photonuclear source file into the system response. This yields a fast and efficient method for analyzing the sensitivity of detector response to photonuclear cross section perturbations.

The results indicate that the photoneutron production from a bremsstrahlung photon source is only mildly sensitive to perturbations in the $(\gamma, n)$ and $(\gamma, 2 n)$ cross sections. For the most extreme perturbations studied here, namely a -0.20 perturbation to both cross sections, the number of neutrons produced in the target decreased by $7-9 \%$ for 10,15 and $20-\mathrm{MeV}$ bremsstrahlung beams respectively. The average energy of the neutrons produced in all of these cases increased by approximately 3-4\% due to the increase in the number of fission reactions. The combination of these two effects leads to an integral detector response change of only $4-8 \%$.

These results apply only to the exact source-target configuration considered here. However, the methodology is general and may be readily applied to any source-target configuration. This has direct implications on the design of active interrogation systems for nuclear nonproliferation and homeland security applications as well as to any other applications using photonuclear cross-section data. For this particular system, the uncertainty in the simulation is on the order of $10 \%$ if the uncertainty in the cross-section data is no greater than $20 \%$.

\section{ACKNOWLEDGMENT}

ORNL is managed and operated for the Department of Energy by UT-Battelle, LLC, under contract DE-AC05-00OR22725. This work was supported in part by the Department of Energy National Nuclear Security Administration NA-241. 


\section{REFERENCES}

1. E. Dupont, I. Raškinyte, A.J. Koning, and D. Ridikas. "Photonuclear Data Evaluations of Actinides up to $130 \mathrm{MeV}$," Proceedings of the International Conference on Nuclear Data for Science and Technology (ND2007), April 21-27, Nice, France (2007).

2. M.-L. Giacri-Mauborgne, D. Ridikas, M.B. Chadwick, P.G. Young and W.B. Wilson. "Photonuclear Physics in Radiation Transport-III: Actinide Cross Sections and Spectra," Nucl. Sci. Eng., 153, 33-40 (2006).

3. J.T. Caldwell, E.T. Dowdy, B.L. Berman, R.A. Alvarez and P. Meyer. "Giant Resonance for Actinide Nuclei: Photoneutron and Photofission Cross Sections of ${ }^{235} \mathrm{U},{ }^{236} \mathrm{U},{ }^{238} \mathrm{U}$ and ${ }^{232}$ Th," Phys. Rev. C, 21(4), 1215-1231 (1980).

4. S. Pozzi, E. Padovani, M. Flaska, and S. Clarke. "MCNP-PoliMi Post-Processing Code Ver. 1.9," Oak Ridge National Laboratory Internal Report, ORNL/TM-2007/33 (2007).

5. Denise Pelowitz (ed.). "MCNPX User's Manual, version 2.5.0," Los Alamos National Laboratory, LA-CP-05-0369 (2005).

6. J.T. Caldwell, E.J. Dowdy, R.A. Alvarez, B.L. Berman and P. Meyer. "Experimental Determination of Photofission and Neutron Multiplicities for ${ }^{235} \mathrm{U},{ }^{236} \mathrm{U},{ }^{238} \mathrm{U}$ and ${ }^{232}$ Th Using Monoenergetic Photons," Nucl. Sci. Eng., 73, 153-163 (1980).

7. Glenn F. Knoll. "Radiation Detection and Measurement, Third Edition" John Wiley \& Sons, Inc., Hoboken, NJ (2000). 

APPENDIX A

MONTE CARLO INTEGRATION ROUTINE 



\section{Monte Carlo Integration Routine}

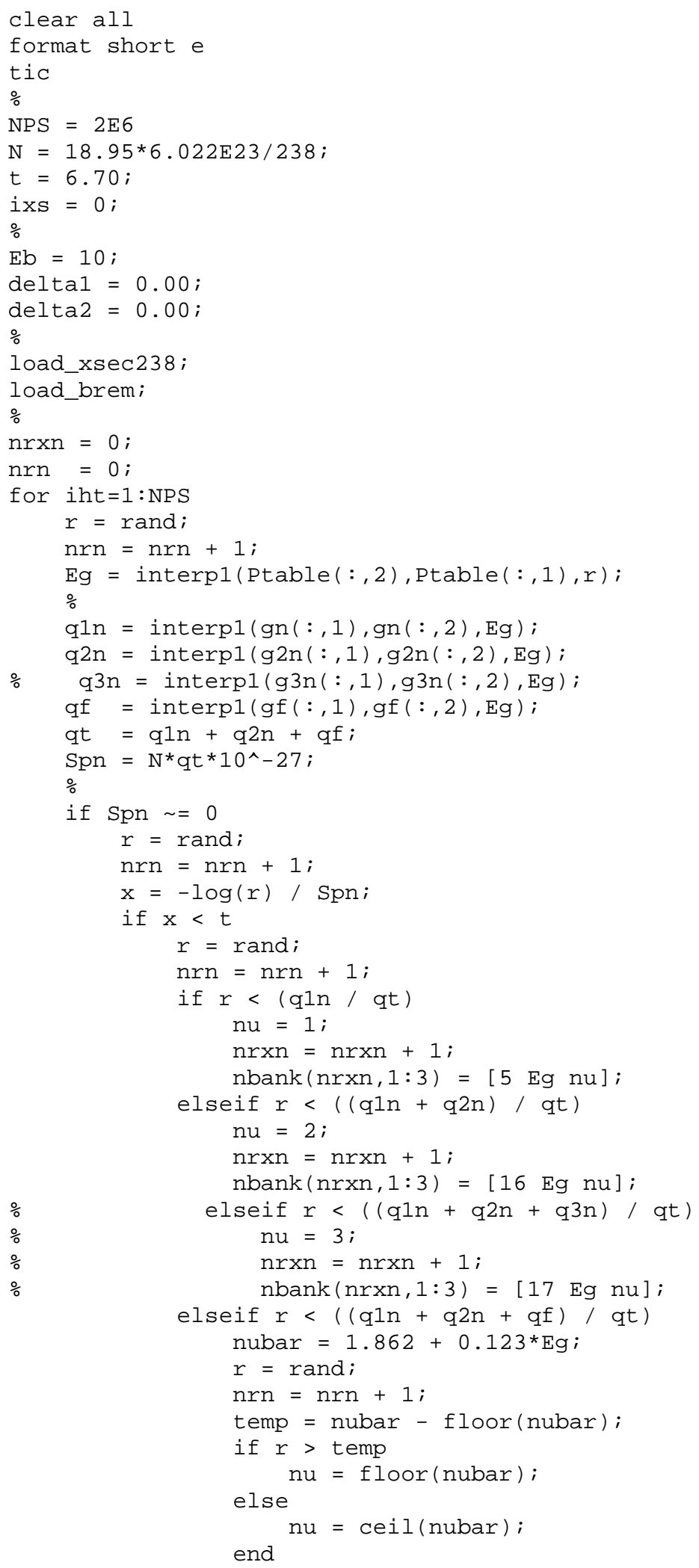




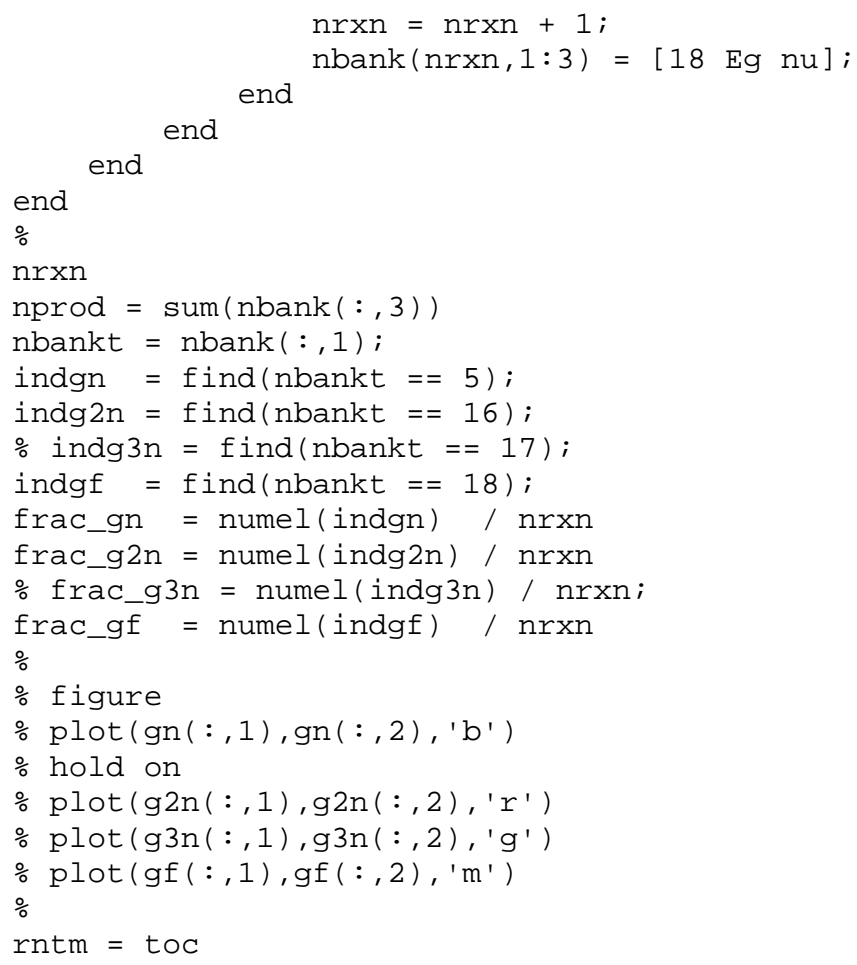


APPENDIX B

MCNP-POLIMI INPUT FILE 



\section{MCNP-PoliMi Input File}

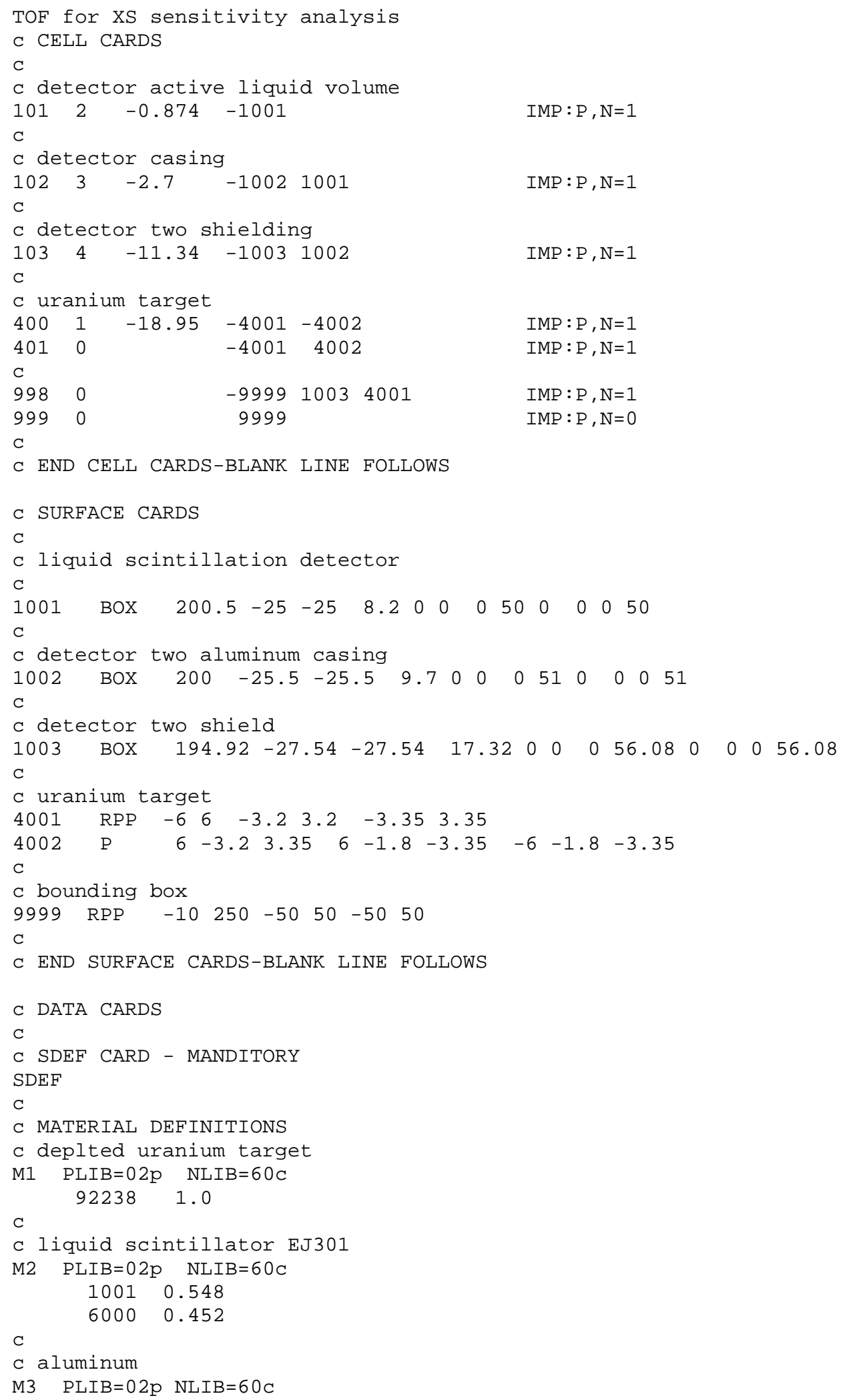




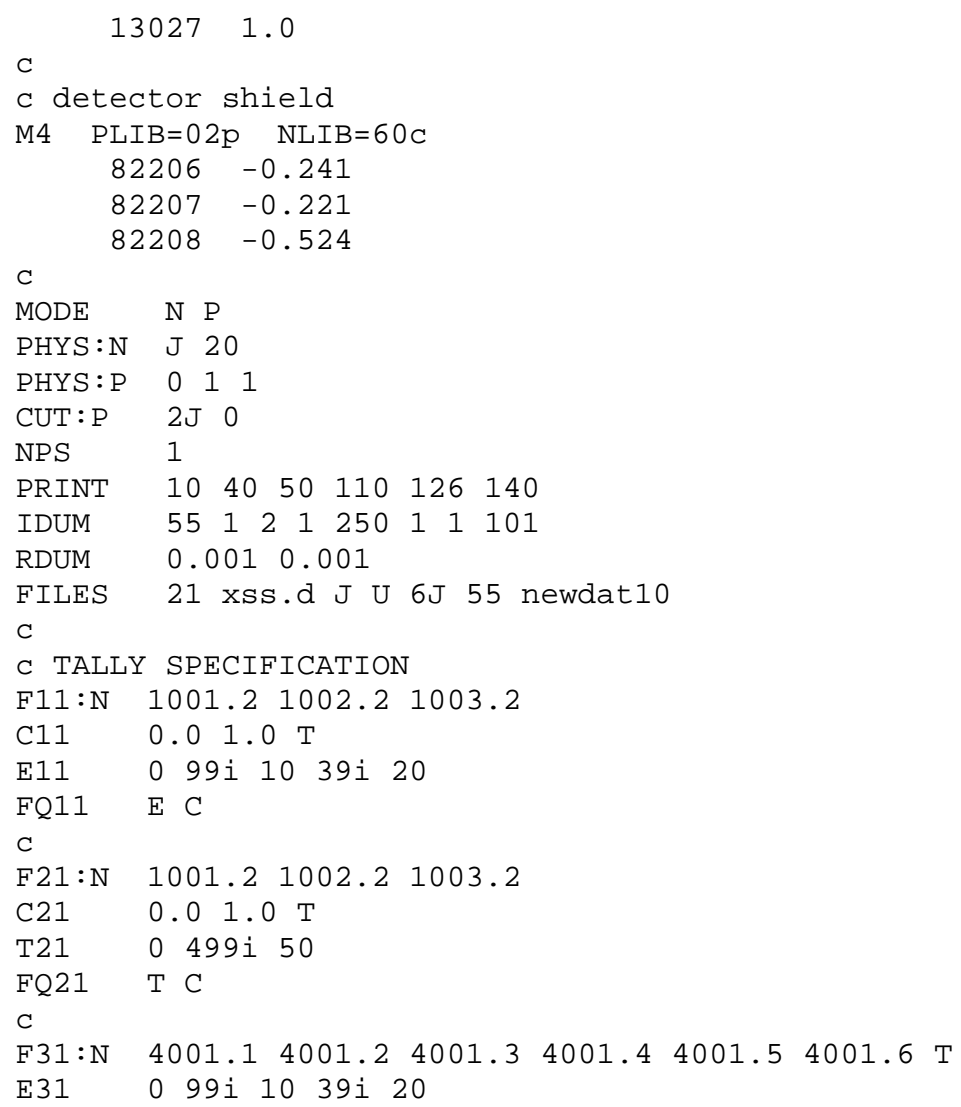

